\title{
Structural Aspects of the Bechgaard Salts and Related Compounds
}

\author{
J.P. Pouget $\left(^{*}\right)$ and S. Ravy \\ Laboratoire de Physique des Solides $\left({ }^{* *}\right)$, Bât. 510, Université Parıs-Sud, 91405 Orsay Cedex, \\ France
}

(Received 11 March 1996, received in final form and accepted 17 June 1996)

PACS.74.70.Kn - Organic superconductors
PACS.71.45.Lr - Charge-density-wave systems
PACS.75.30.Fv - Spin-density waves

Abstract. - Structural aspects of the Bechgaard salts (TMTSF) ${ }_{2} \mathrm{X}$ and related 2:1 salts. mostly based on the sulfur donors TMTTF and BCPTTF as well as on the hybrid TMDTDSF donor, are considered in relationship with their physical properties. The basic structure of these salts is reviewed with a special emphasize on the dimerization of the organic stack and on the disorder of the anıons $(\mathrm{X})$. Then the density waves instabilities driven by the donor stacks are discussed. It is shown in particular that (TMTSF) ${ }_{2} \mathrm{PF}_{6}$ exhibits a mixed $2 k_{\mathrm{F}}$ CDW-SDW ground state. The $4 k_{\mathrm{F}}$ charge localization phenomena and the $2 k_{\mathrm{F}}$ instability of the organic stacks are described in relationship with the structural dimerization and the importance of Coulomb interactions. In the strong charge localization case the spin Peierls instabilities of $(\mathrm{TMTTF})_{2} \mathrm{PF}_{6}$ and of (BCPTTF $)_{2} \mathrm{PF}_{6}$ are more particularly detailed. General aspects of the anıon orderıng transitions exhibited by salts with non centrosymmetrical anıons are pointed out as well as the influence of such transitions on the electronic properties. Finally the relevance of these structural features is briefly discussed by comparing the Bechgaard salts series' of quasi-one dimensional conductors to the family of two dimensional (BEDTTTF $)_{2} \mathrm{X}$ conductors.

\section{Introduction}

The first organıc material exhibiting conducting properties was prepared in 1954 by exposing the perylene molecule to bromine. However it was only with the synthesis of the electron acceptor TCNQ (tetracyanoquinodimethane) in 1960 and the finding of a room temperature electrical conductivity of $\sim 100 \mathrm{~S} / \mathrm{cm}$ in the charge transfer salts Qn-(TCNQ) 2 and NMPTCNQ that the field of organic conductors really opened. One of the main pioneers of this field was Schegolev and his school [1]. Then the synthesis of the electron donor TTF (tetrathiafulvalene) in 1972 and the report at that time of a high increase of conductivity down to $60 \mathrm{~K}$ in TTF-TCNQ was at the origin of a world wide interest for the organic conductors. In these first generation charge transfer salts the metallic properties could not be maintained down to low temperature because of the occurrence of a Peierls metal-insulator phase transition. In such one dimensional (1D) conductors this transition is driven by the instability of the electron gas

$\left.{ }^{*}\right)$ Author for correspondence (e-mall: pouget@lps.u-psud.fr)

$\left({ }^{*}\right)$ (CNRS URA 2)

(C) Les Éditions de Physıque 1996 
towards the formation of charge density waves (CDW) at the critical wave vector $2 k_{\mathbf{F}}\left(k_{\mathbf{F}}\right.$ is the Fermi wave vector of the 1D electron gas) [2-4]. A real break through occurred en 1980 with the stabilization of the metallic state under pressure and the discovery of organic superconductivity at $T_{\mathrm{s}} \sim 1 \mathrm{~K}$ in (TMTSF) ${ }_{2} \mathrm{PF}_{6}$, which is based on the tetramethyltetraselenafulvalene donor. With different monovalent anions $\mathrm{X}$ such as $\mathrm{PF}_{6}, \mathrm{ClO}_{4}, \mathrm{ReO}_{4}, \mathrm{NO}_{3} \ldots$ a whole family of quasi-1D conductors, now known as the Bechgaard salts, and which present an impressive number of novel physical features and new electronic ground states, were synthesized [3,5]. A major advance was also made, in the middle of the 80 's, with the synthesis of $2 \mathrm{D}$ organic conductors based on the TTF derivative BEDTTTF [bis (ethylenedithio) tetrathiafulvalene] which exhibited a $T_{\mathrm{s}}$ approaching and even overcoming the " $10 \mathrm{~K}$ barrier" in $\beta$-(BEDTTTF $)_{2} I_{3}$ and $\kappa-(\mathrm{BEDTTTF})_{2} \mathrm{Cu}(\mathrm{SCN})_{2}$ and $\mathrm{Cu} \mathrm{N}(\mathrm{CN})_{2} \mathrm{Br}[5,6]$. Up to now the superconductivity is found in more than 50 organic compounds. Two others important families of organic superconductors are the quast-1D salts based on the metal-organic acceptor complexes $\mathrm{M}(\mathrm{dmit})_{2}(\mathrm{M}=\mathrm{Ni}, \mathrm{Pd})$, with a highest $T_{\mathrm{s}}$ of $6.5 \mathrm{~K}[5,7]$, and the $3 \mathrm{D}$ alkali-metal $\mathrm{C}_{60}$ fullerides exhibiting a $T_{\mathrm{s}}$ as high as $32 \mathrm{~K}$ in $\mathrm{Rb}_{2} \mathrm{CsC}_{60}$ [8].

Because of the instability of the 1D electron gas towards the formation of $2 k_{\mathrm{F}} \mathrm{CDW}$ or $2 k_{\mathrm{F}}$ spın density waves (SDW) or even, in presence of strong electron-electron repulsions, towards the formation of $4 k_{\mathrm{F}} \mathrm{CDW}$, very complex phase diagrams are exhibited by the quasi$1 \mathrm{D}$ organic charge transfert salts. Due to the presence, in these "soft" organic salts, of a $1^{\text {st }}$ order electron-phonon coupling the $2 k_{\mathrm{F}}$ and $4 k_{\mathrm{F}}$ electronic CDW instabilities are accompanied by a modulation of the molecular positions at the same critical wave vector. These structures are also, because of the divergence of the response function of the electronic degrees of freedom, very sensitive to various kinds of disorder. This is particularly the case of structural disorders associated to orientational degrees of freedom of the anion X (in the case of the Bechgaard salts) or to conformational degrees of freedom of the organic molecule (in the case of the ethylene groups of the BEDTTTF molecules). The collective ordering of such degrees of freedom can also lead to substantial modifications of the electronic phase diagram. In all these cases there is a subtle interplay between the order-disorder effects and the electronic properties of the organic salts [9].

In this paper we present a short review of these structural aspects in the (TMTSF) 2 X family of quasi-1D conductors and its donor derivatives where the prototype crystallographic structure of the Bechgaard salts is kept. The basic aspects of this structure are considered Section 2. The instabilities exhibited by the metallic organic stack and its ground states will be analysed Section 3 . The transitions related to anion ordering effects will be discussed Section 4 . Finally some concluding remarks will be done Section 5 .

\section{Basic Structure of the Bechgaard Salts}

2.1. The Prototype Structure. - These salts crystallize in the triclinic $\mathrm{P} \overline{\mathrm{I}}$ space group with two donors and one anion $\mathrm{X}$ per unit cell [10]. Figures $1 \mathrm{a}$ and $\mathrm{b}$ show two projections of the structure of (TMTSF) $)_{2} \mathrm{X}$. The TMTSF donors are related by inversion symmetry with inversion centers located between the molecules. They form $(\mathbf{a}, \mathbf{b})$ layers which alternate with the anions $\mathrm{X}$ along the $c$ direction. In the direction (a) of highest conductivity the molecules form zig-zag stacks which are slightly dimerized (a feature which will be discussed more explicitly in Sect. 2.2). The anıons are placed in centrosymmetrical cavities delimited by the methyl groups of the TMTSF molecules. The location of non centrosymmetrical anions in these cavities introduces a substantial disorder in the structure, which will be more explicitly considered in Section 2.3. 


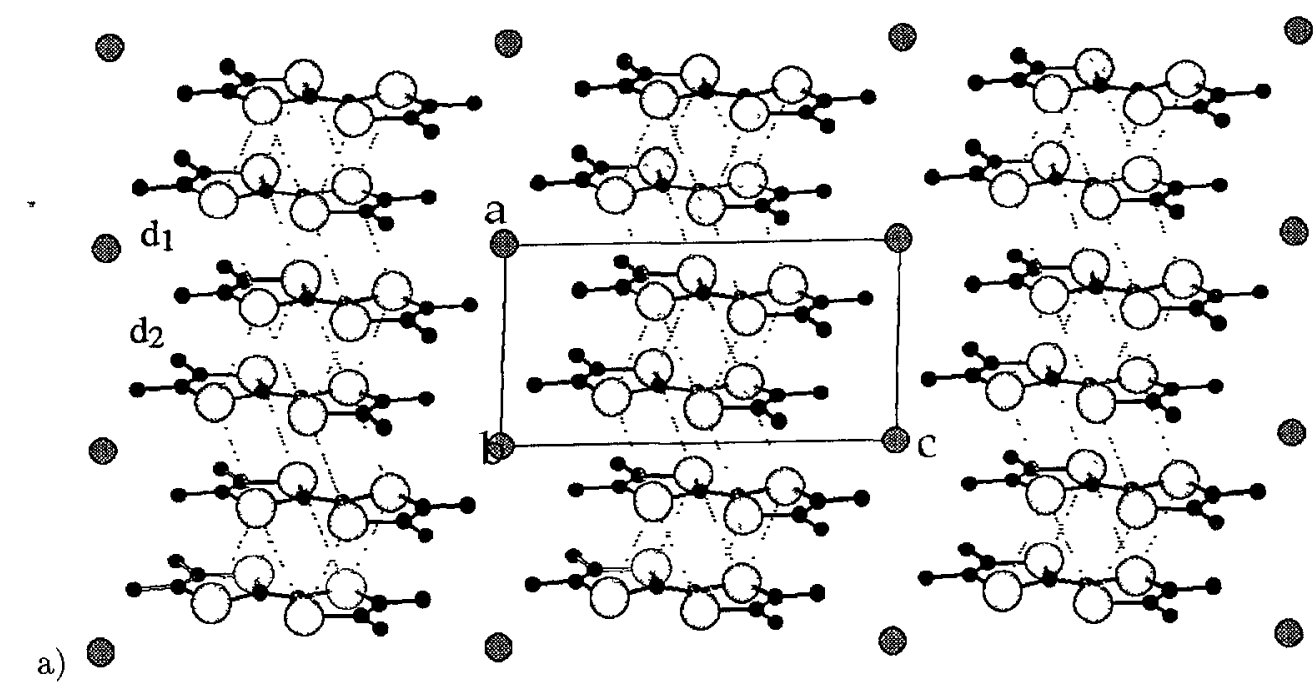

6

중<smiles>CC1OC(C2OC(C)C(C)C2O)C(O)C(C)C1C</smiles><smiles>C[C@@H]1OC(C2OC[C@@H](C)[C@H](C)O2)O[C@H]1C</smiles>

(2)

궁

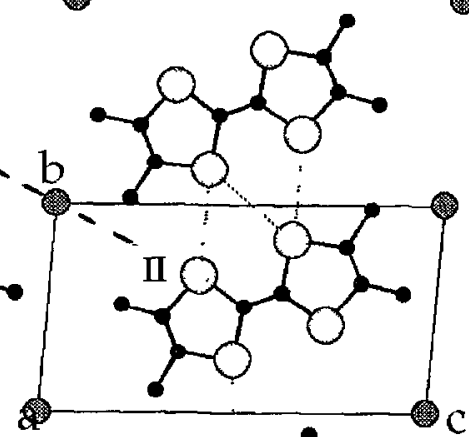

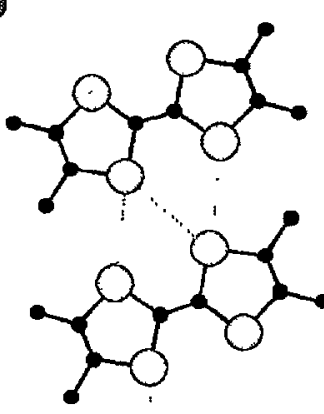

b)

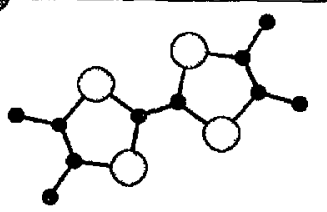<smiles>C[C@H]1COC(C2OC[C@@H](C)[C@@H](C)O2)O[C@H]1C</smiles>

Fig. 1. - Projections of the crystallographic structure of (TMTSF) $)_{2} \mathrm{X}$ in the (a.c) plane (a) and the (b,c) plane (b). Only the Se (large grey dots) and C (small black dots) atoms of the TMTSF molecule are represented. The grey dots of medium size between the molecules symbolizes the location of the anions X.

The (TMTTF $)_{2} \mathrm{X}$ salts built on the $\mathrm{S}$ analogue TMTTF (tetramethyltetrathiafulvalene) donor are isostructural to the Bechgaard salts. In the case of (TMDTDSF) 2 X salts, where the TMDTDSF molecule is a hybrid of the TMTTF and TMTSF molecules, there is a random orientation of the non-centrosymmetrical TMDTDSF molecule in the structure [11]. In these latest salts the $\mathrm{P} \overline{1}$ symmetry is only kept on average. In others 2:1 salts made with unsymmetrical donors such as t-TTF (trimethyl Tetra Thia Fulvalene), DMET (a hybrid of TMTSF and BEDTTTF) or BCPTTF (Benzo Cyclo Pentyl Tetra Thia Fulvalene) the molecules alternate in stacking direction, which keeps the inversion symmetry. At the difference of (TMDTDSF) 2 $\mathrm{X}$ these $\mathrm{P} \overline{1}$ salts are not disordered. 
Table I. - Intrachain transfer integrals, $t_{\mathrm{s} 1}$ and $t_{\mathrm{s} 2}$, and degree of dimerization, $\Delta t_{\mathrm{s}} /\left\langle t_{\mathrm{s}}\right\rangle$. of some $(\text { TMTSF })_{2} X$, (TMTTF $)_{2} \mathrm{X}$ and $\left(B C P T T F_{2}\right) X$ salts calculated with single $\zeta$ Slater type orbitals (from Ref. [13]).

\begin{tabular}{|c|c|c|c|c|c|}
\hline Compound & conditions & $t_{\mathrm{s} 1}(\mathrm{meV})$ & $t_{\mathrm{s} 2}(\mathrm{meV})$ & $\Delta t_{\mathrm{s}} /\left\langle t_{\mathrm{s}}\right\rangle$ & Reference \\
\hline \multirow{3}{*}{$(\mathrm{TMTSF})_{2} \mathrm{PF}_{6}$} & $295 \mathrm{~K}, 1 \mathrm{bar}$ & 252 & 209 & 0.19 & \multirow{3}{*}{ (a) } \\
\hline & $4 \mathrm{~K}, 1$ bar & 280 & 254 & 0.10 & \\
\hline & $1.7 \mathrm{~K}, 7 \mathrm{kbars}$ & 290 & 272 & 0.06 & \\
\hline \multirow[t]{2}{*}{$(\mathrm{TMTSF})_{2} \mathrm{ClO}_{4}$} & $295 \mathrm{~K}, 1 \mathrm{bar}$ & 258 & 221 & 0.15 & \multirow[t]{2}{*}{ (a) } \\
\hline & $7 \mathrm{~K}, 1 \mathrm{bar}$ & 287 & 266 & 0.08 & \\
\hline$(\mathrm{TMTSF})_{2} \mathrm{ReO}_{4}$ & $295 \mathrm{~K}, 1 \mathrm{bar}$ & 248 & 215 & 0.14 & (b) \\
\hline$(\mathrm{TMTSF})_{2} \mathrm{NO}_{3}$ & $295 \mathrm{~K}, 1$ bar & 243 & 224 & 0.08 & (c) \\
\hline \multirow[t]{2}{*}{$(\mathrm{TMTTF})_{2} \mathrm{PF}_{6}$} & $295 \mathrm{~K}, 1$ bar & 137 & 93 & 0.38 & \multirow[t]{2}{*}{ (a) } \\
\hline & $4 \mathrm{~K}, 1$ bar & 146 & 123 & 0.17 & \\
\hline$(\mathrm{TMTTF})_{2} \mathrm{ClO}_{4}$ & $295 \mathrm{~K} .1 \mathrm{bar}$ & 140 & 100 & 0.33 & (b) \\
\hline$(\mathrm{TMTTF})_{2} \mathrm{Br}$ & $295 \mathrm{~K}, 1 \mathrm{bar}$ & 133 & 119 & 0.11 & (a) \\
\hline$(\mathrm{BCPTTF})_{2} \mathrm{PF}_{6}$ & $295 \mathrm{~K}, 1 \mathrm{bar}$ & 118 & 79 & 0.40 & (d) \\
\hline
\end{tabular}

[(TMTSF $)_{1-x}\left(\mathrm{TMTTF}_{x}\right]_{2} \mathrm{X}$ solid solutions can be also formed. However in the case of the $\mathrm{X}=\mathrm{ReO}_{4}$ solid solution an alternate order of the TMTSF and TMTTF molecules along the stacking direction, $\mathbf{a}$, as well as in the $\mathbf{b}$ and $\mathbf{c}$ directions is found for $x \sim 0.5$ [12]. This molecular orderıng, which doubles the $b$ and $c$ lattice parameters, now breaks the inversion symmetry of the prototype structure of the Bechgaard salts. This ordered structure however has not been completely refined.

2.2. The Dimerization of the Organic Stack. - At the difference of TTF-TCNQ or Qn-(TCNQ) $)_{2}$ the stacks of organic molecules of the Bechgaard salts and related compounds are slightly dimerized. Such a dimerization can have very drastic consequences for the electronic properties of these $2: 1$ salts because, as the reciprocal periodicity $2 \pi / a$ of the stack corresponds to the $4 k_{\mathrm{F}}$ critical wave vector of the $1 \mathrm{D}$ correlated electron gas, the lat tice effects can enhance the charge localization phenomena which will be considered in Section 3.2.

In the literature the dimerization of the zig-zag stack is often quantified by the difference of distances between the average molecular planes along a $\left(2\left(d_{2}-d_{1}\right) / a \sim 0.8 \%\right.$ in $\left.(\mathrm{TMTSF})_{2} \mathrm{PF}_{6}\right)$. However such an analysis ignores that a significant dimerization can be also produced by a shift of the molecules transverse to the stack direction. As both kinds of displacement can comparably affect the intrastack electronic interactions, the dimerization can be better quantified by the calculation of intermolecular transfer integrals along the $d_{1}\left(t_{\mathrm{s} 1}\right)$ and $d_{2}\left(t_{\mathrm{s} 2}\right)$ bonds defined Figure 1a. Table I gives these $t_{\mathrm{s} 2}$ for the donor's HOMO in the framework of the extended Hückel theory when the anonic potential is neglected [13]. The relative difference $2\left(t_{\mathrm{s} 1}-t_{\mathrm{s} 2}\right) /\left(t_{\mathrm{s} 1}+t_{\mathrm{s} 2}\right) \equiv \Delta t_{\mathrm{s}} /\left\langle t_{\mathrm{s}}\right\rangle$ allows to define the degree of dimerization of the stack. Table I shows in particular that the dimerization:

- increases from the "Se" to the "S" donors: TMTSF $\rightarrow$ TMTTF, BCPTTF,

- decreases when the lattice contracts (i.e upon cooling or under pressure),

- varies with the shape of the anion (.$e$ decreases along the sequence $\mathrm{PF}_{6} \rightarrow \mathrm{ClO}_{4}, \mathrm{ReO}_{4} \rightarrow$ $\mathrm{NO}_{3}, \mathrm{Br}$ ). 
The non regularity of the organic columns seems to be caused by steric constraints exercised by the anions on the donor layers. In this respect an enhanced dimerization is observed in TMTSF and TMTTF salts with large anions such as $\mathrm{X}=\mathrm{Re}_{6} \mathrm{Se}_{5} \mathrm{Cl}_{9}$ [14].

Figure 1a shows that the anions face the bonds where the $d_{1}$ intermolecular distance is the shortest. This implies that along the stack the " $d_{1}$ dimers", where the $\mathrm{p}_{\pi}$ conduction electron density is enhanced, experience a stronger Coulomb repulsion from the anions than the " $d_{2}$ interdimers" bonds. The decrease of the dimerization found upon cooling or under pressure will thus reduce the Coulomb repulsions between the $\mathrm{p}_{\pi}$ electrons and the anionic sublattice.

2.3. The AnION Disorder. - In the Bechgaard salts the methyl groups of the organic TMTSF or TMTTF molecules delimit centrosymmetrical cavities where the anions are located. The various anions which thus can be accommodated in such cavities can be classified according to their symmetry: spherical $\left(\mathrm{Br}^{-}\right)$, octahedral $\left(\mathrm{PF}_{6}^{-}, \mathrm{AsF}_{6}^{-}, \mathrm{SbF}_{6}^{-}, \ldots\right)$, tetrahedral $\left(\mathrm{ClO}_{4}^{-}\right.$, $\left.\mathrm{BF}_{4}^{-}, \mathrm{ReO}_{4}^{-}, \ldots\right)$, triangular $\left(\mathrm{NO}_{3}^{-}\right)$or linear $\left(\mathrm{SCN}^{-}\right)$. The avalable structural data show that these anrons stand on inversion centers at least at room temperature (RT). In the case of centrosymmetric (spherical or octahedral) anions this location does not introduce any disorder in the structure. In the case of non centrosymmetric (tetrahedral, triangular or linear) anions the resulting conflict is generally considered to imply some sort of structural disorder with two (or more) orientations of the anions so that the inversion centers are present on average only. In these salts an orientational ordering of the anions generally occurs at low temperature. It is achieved by the formation of superstructures through structural phase transitions which will be discussed more explicitly in Section 4 . Such anıon ordering transitions affect sizeably the electronic structure.

The RT anion disorder seems to be special to the (TMTSF) $)_{2} \mathrm{X}$ and (TMTTF) ${ }_{2} \mathrm{X}$ families. In others compounds built with different donors the non centrosymmetric anions are found to be already ordered at RT. For example it is found a staggered orientation of the tetrahedral anons which doubles the $a$ lattice perodicity in (t-TTF) ${ }_{2} \mathrm{ClO}_{4}$ and $\mathrm{BF}_{4}$ and the $b$ and $c$ lattice periodicities in (BEDTTTF) ${ }_{2} \mathrm{ReO}_{4}$. The RT orientational disorder seems to be the signature of a weak anion-donor interactions, probably due to the location of the anions in well isolated cavities formed by the methyl groups of the TMTSF and TMTTF molecules.

The outer ( $\mathrm{O}$ or $\mathrm{F}$ ) atoms of the anion tend to establish contacts (i.e. bonds) with the $\mathrm{S}$ or Se atoms of the two inversion symmetry related organic molecules (labelled I and II in Fig. 1b). Such contacts determine the orientation of the anion in the cavity (Fig. 2). There are two contacts in the case of the $\mathrm{PF}_{6}^{-}$octahedral anion, one contact in the case of the $\mathrm{ClO}_{4}^{-}$ or $\mathrm{ReO}_{4}^{-}$tetrahedral aniou and nearly no contact in the case of small anions such as $\mathrm{Br}^{-}$or $\mathrm{NO}_{3}^{-}$. The degree of dimerization of the organic stack apparently increases with the number of such contacts (Tab. I).

\section{The Donor Stack Instabilities and Associated Ground States}

3.1. The BechgaArd Salts. - The (TMTSF) $)_{2} \mathrm{X}$ salts are quasi-1D conductors. Optical conductivity shows that at RT the electronic transport is coherent only along the stack direction $[3,5]$. Figure 3 gives a schematic representation of the donor's HOMO electronic dispersion in this direction. The band structure is folded at $\pm \pi / a$ because of the stack dimerization. At the Brillouin zones boundaries the gap $2 \Delta_{\mathrm{D}}^{b}$ is due to the inequivalence between the $d_{1}$ and $d_{2}$ bonds (all the molecular sites, which are related by inversion symmetry, are equivalent). In the framework of the tight binding description of the band structure one has:

$$
\Delta_{\mathrm{D}}^{\mathrm{b}}=\left|t_{\mathrm{s} 1}-t_{\mathrm{s} 2}+V_{1}-V_{2}\right|
$$



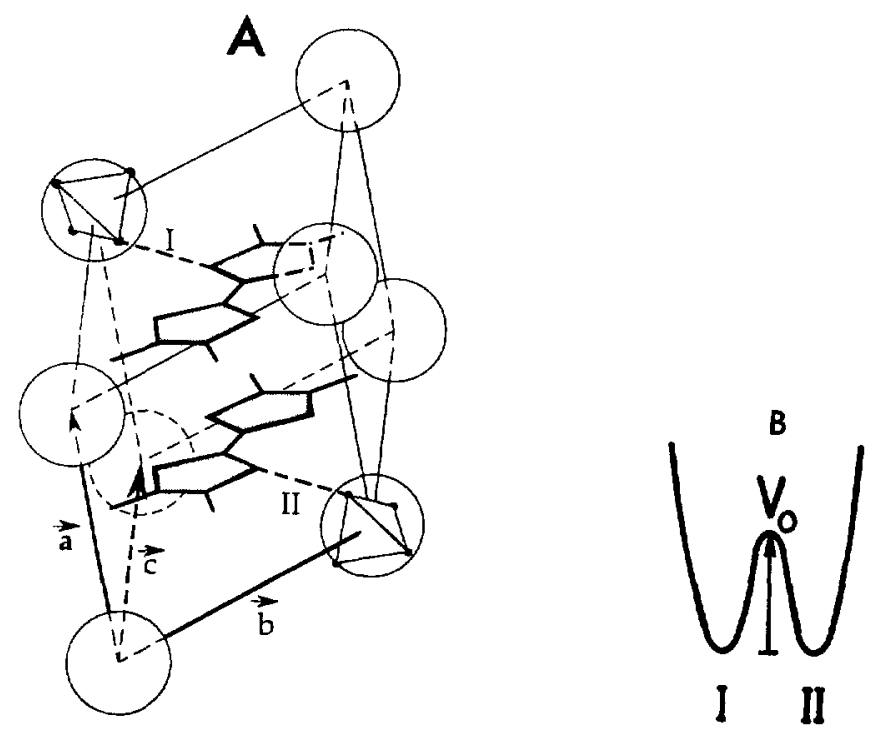

Fig. 2. - Two possible orientations of a tetrahedral anıon $X$ in a Bechgaard salt (a) and schematical representation of the potential experienced by such an anion from its surrounding (b). I and II denotes the two inversion symmetry related onentations of the anion.

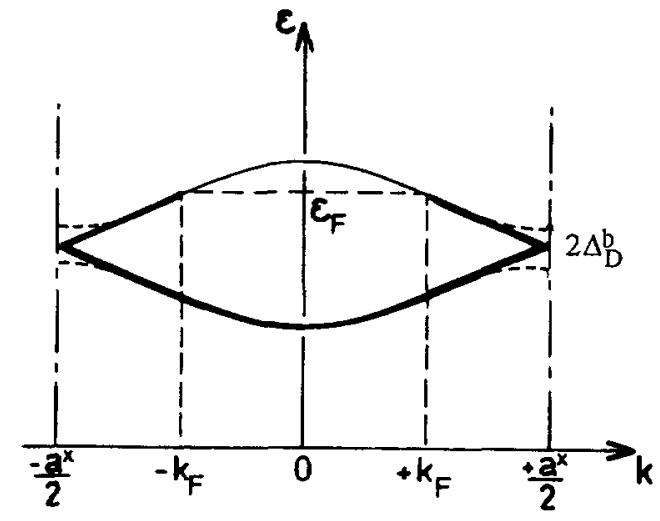

Fig. 3. - Schematic representation of the one electron band dispersion of the Bechgaard salts in chain direction. The dashed line shows the effect of the dimernzation of the organic stack on the band structure.

where $t_{s 1}\left(t_{\mathrm{s} 2}\right)$ and $V_{1}\left(V_{2}\right)$ are the intra (inter) dimer transfer integrals and $d_{1}\left(d_{2}\right)$ bond potentials (mostly due to anions) respectively. The transfer of one electron to each anon leaves 0.5 hole on average per donor. In absence of dimerization the conduction band will be $3 / 4$ filled. With the formation of one dimer per chain repeat unit, giving rise to two HOMO like bands per Brilloum zone, the upper band is half filled (i.e $k_{\mathrm{F}}= \pm \pi / 2 a$ in Fig. 3). In this case the critical wave vector $2 k_{\mathrm{F}}$ of the CDW or SDW instability is at $\pi / a$. Interchain coupling effects $\left(t_{\perp}\right)$ become relevant at low temperature [15]. However the anisotropy of transfer integrals is large enough $\left(t_{\perp} /\left(t_{\mathrm{s}}\right\rangle \sim 1 / 10\right)$ so that the warped Fermı surface remains opened. 


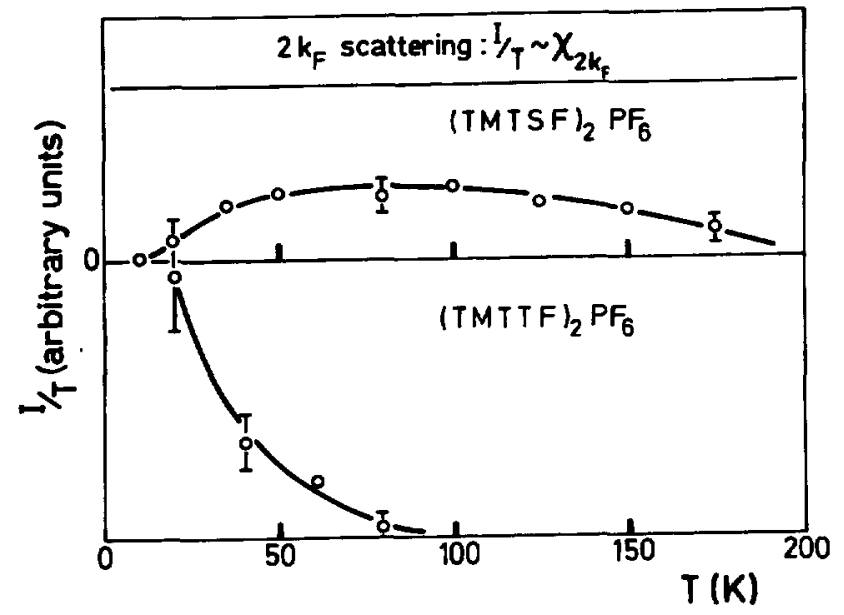

Fig. 4. - Temperature dependence of the $2 k_{\mathrm{F}} \mathrm{CDW}$ (top) and spin Peierls (bottom) response functions of $(\mathrm{TMTSF})_{2} \mathrm{PF}_{6}$ and $(\mathrm{TMTTF})_{2} \mathrm{PF}_{6}$ respectively.

3.1.1. $2 k_{\mathrm{F}} C D W$ Fluctuations. - $\mathrm{X}$-ray diffuse scattering investigations of (TMTSF) ${ }_{2} \mathrm{PF}_{6}$ and $\mathrm{AsF}_{6}[16]$ reveal below about $175-150 \mathrm{~K}$ the presence of weak $2 k_{\mathrm{F}}$ diffuse lines at large scattering wave vectors $\left(Q_{\|} \sim 2.5-4.5 a^{*}\right)$ in the reciprocal space. Such a diffuse scattering, already observed in others TMTSF charge transfer salts, like TMTSF-DMTCNQ [17], is the signature of a displacive instability of the TMTSF stack driven by the $2 k_{\mathrm{F}}$ CDW instability of its 1D electron gas. However at the difference of TMTSF-DMTCNQ the $2 k_{\mathrm{F}}$ scattering of (TMTSF) ${ }_{2} \mathrm{PF}_{6}$ and $\mathrm{AsF}_{6}$ does not exhibit a critical behavior leading to a CDW Peierls ground state upon cooling down. The intrachain CDW correlation length remains limited at $\sim 10 \AA$ (slightly more than the chain periodicity $a=7.3 \AA$ ) and the $2 k_{\mathrm{F}} \mathrm{X}$-ray diffuse scattering peak intensity, even corrected by the thermal population factor $(I / T)$, decreases below about $50 \mathrm{~K}$ and vanishes at low temperature (Fig. 4). It is interesting to remark that the $1 \mathrm{D} 2 k_{\mathrm{F}}$ structural fluctuations vanish in the temperature range where a $2 \mathrm{D}$ or $3 \mathrm{D}$ regime of critical SDW fluctuations, probed by NMR [18], builts up. However between about $20 \mathrm{~K}$ and $150 \mathrm{~K}$ the $2 k_{\mathrm{F}}$ CDW and SDW fluctuations coexist. The non observation of $2 k_{\mathrm{F}}$ structural fluctuations below $20 \mathrm{~K}$ does not necessarly mean that the electronıc CDW response function vanishes. It could be that the electronic instability becomes decoupled from the lattice degrees of freedom. In the next part we shall see that there are evidences of a survival of the electronic CDW below $T_{\mathrm{N}}$ in (TMTSF) ${ }_{2} \mathrm{PF}_{6}$.

3.1.2. The Ground State of (TMTSF $)_{2} \mathrm{PF}_{6}$. - (TMTSF $)_{2} \mathrm{PF}_{6}$ undergoes at $T_{\mathrm{N}}=12 \mathrm{~K}$ a metalinsulator phase transition which suggests the occurrence of a $2 k_{\mathrm{F}}$ density wave ground state. The spin susceptibility anisotropy [19], the observation of an antiferromagnetic resonance [20] and the measurement of a local field by NMR and $\mu$ SR [21] prove the magnetic nature of this ground state. The magnetic perodicity has not yet been detected by elastic neutron scattering. However two independent proton NMR experiments suggest a sinusoidal SDW modulation of wave vector $\mathbf{q S D W}=\left(0.5,0.20-0.24,{ }^{7}\right)$ and of amplitude $0.08 \mu_{\mathrm{B}}$ [22]. This incommensurate SDW collectively slides under the action of an electric field [23].

The metal-insulator phase transition of (TMTSF) ${ }_{2} \mathrm{PF}_{6}$ can be caused [5] by the nesting of its Fermi surface by the qSDW wave vector, with a gap opening due to the onset of a periodic $2 k_{\mathrm{F}}$ exchange potential, as predicted long time ago by Slater [24]. This mechanism, analogous 


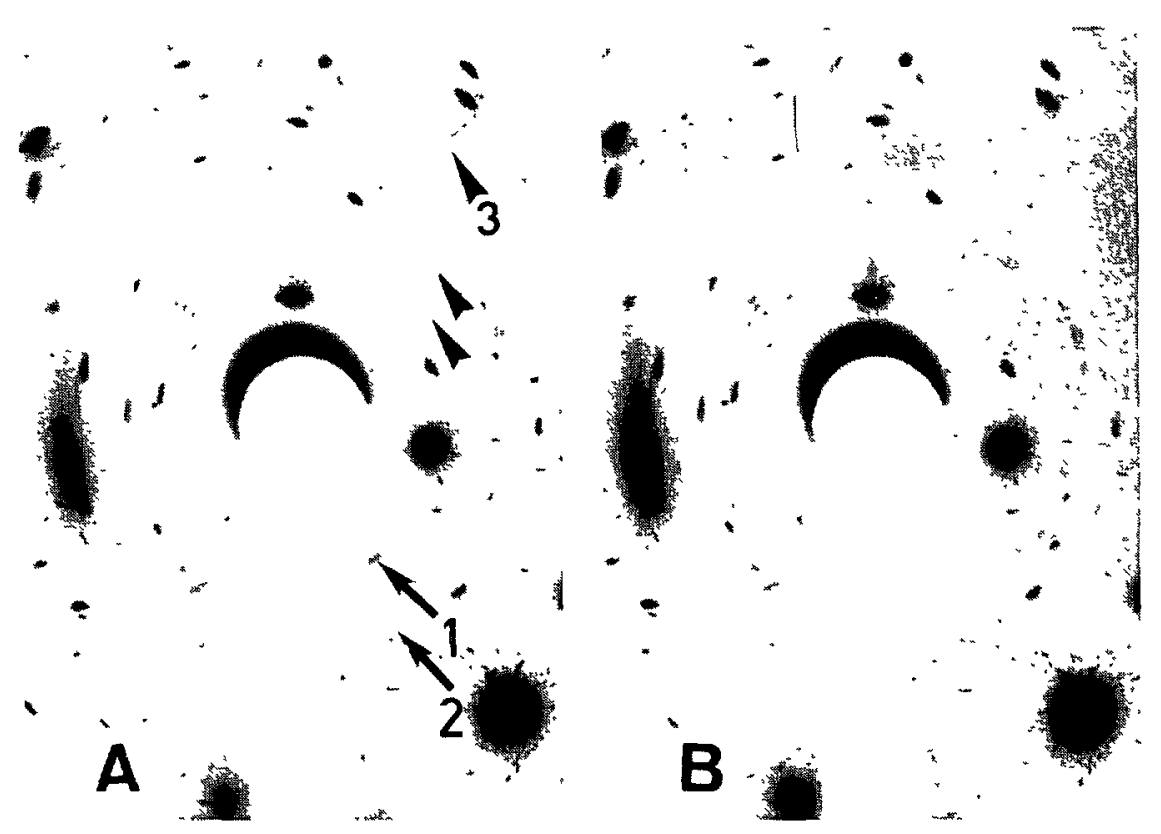

Fig. 5. - X-ray patterns from (TMTSF) ${ }_{2} \mathrm{PF}_{6}$ at $10.7 \mathrm{~K}$ (a) and $15.5 \mathrm{~K}$ (b). In (a) the long (short) arrows point towards the $\mathbf{q}_{1}\left(\mathbf{q}_{2}\right)$ satellite reflections. The a axis is horizontal. The coordinates of the satellites labelled 1,2 and 3 in (a) are $(0.5, \overline{1.25}, \overline{1.25}),(0.5, \overline{1.75}, \overline{1.75})$ and $(1,2.5, \overline{1.5})$ respectively. with uncertainties of 0.05 along the $h$ and $k$ components and of 0.2 along the $\ell$ component. These $\mathrm{X}$-ray patterns have been taken with the CuK $\alpha(\lambda=1.542 \AA)$ radiation without $\lambda / 2$ contamination $(15 \mathrm{kV}, 40 \mathrm{~mA})$ and with an exposure time of 32 hours.

to that of the Peierls transition, must lead to a $2^{\text {nd }}$ order phase transition. However the thermal behavior of the electrical resistivity [25] and of the internal field [21] just below $T_{\mathrm{N}}$ suggests more likely a weak $1^{\text {st }}$ order phase transition. A $1^{\text {st }}$ order magnetic transition generally occurs in presence of a sizeable magnetoelastic coupling. A possible coupling between the electronic degrees of freedom and the lattice has been also invoked to explain the dynamical mass enhancement of the SDW condensate [23] and the large configuration entropy found by specific heat measurements [26] below $T_{\mathrm{N}}$. In the purpose to detect a possible coupling between the SDW and the lattice we have performed an X-ray diffuse scattering investigation of (TMTSF) ${ }_{2} \mathrm{PF}_{6}$ below $T_{\mathrm{N}}$.

Figure 5 shows two X-ray patterns taken (a) below $T_{\mathrm{N}}$ and (b) above $T_{\mathrm{N}}$. Five weak and

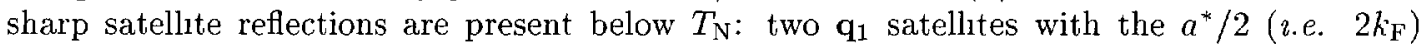
wave vector component and three $\mathbf{q}_{2}$ satellites with the $a^{*}$ wave vector component. Within experimental errors one has $2 \mathbf{q}_{1}=\mathbf{q}_{2}$. A computer indexation of the $\mathrm{X}$-ray pattern of Figure $5 \mathrm{a}$ gives the following components for the $\mathbf{q}_{1}$ reduced wave vector:

$$
\begin{aligned}
& q_{1}^{a}=0.50 \pm 0.05 a^{*} \\
& q_{1}^{b}=0.25 \pm 0.05 b^{*} \\
& q_{1}^{c}=0.25 \pm 0.20 c^{*}
\end{aligned}
$$

Within experımental errors $\mathbf{q}_{1}$ corresponds to $\mathbf{q}_{\mathrm{SDW}}$ determıned by NMR. These satellites reflections disappear at $13.25 \pm 0.25 \mathrm{~K}$, which corresponds to the $T_{\mathrm{N}}$ of slightly irradiated samples [25]. Such satellites were also detected on two others (TMTSF) ${ }_{2} \mathrm{PF}_{6}$ samples (one $\mathbf{q}_{2}$ 
satellite on a first sample, four $\mathbf{q}_{1}$ and two $\mathbf{q}_{2}$ satellites on a second sample). The satellite reflections are always observed near the origin of the reciprocal space. No $\mathbf{q}_{1}$ satellite reflection is observed at the location of the high temperature $2 k_{\mathrm{F}}$ diffuse lines reported in Section 3.1.1. This means that their structure factor is not that of a diplacive modulation of the organic stacks.

The $\mathbf{q}_{1}$ and $\mathbf{q}_{2}$ satellite reflections are of very weak intensity. One of the strongest satellites (labelled (1) in Fig. 5a) has about $10^{-5}$ times the intensity of the (10) ) main Bragg reflection. The $\mathbf{q}_{1}$ and $\mathbf{q}_{2}$ satellites labelled (2) and (3) in Figure $5 a$ are respectively two and three times less intense. These satellite intensities are however much stronger than the intensity expected from the X-ray magnetic scattering by the $2 k_{\mathrm{F}} \mathrm{SDW}$, for which one can estimate that $I_{\mathrm{SDW}} / I_{\mathrm{Bragg}} \sim 10^{-10}[28]$. The weakness of the $\mathbf{q}_{1}$ satellite intensity and the location of these satellites near the origin of the reciprocal space suggest that we could have detected a purely electronic CDW. From the intensity of the satellites (1) and (2) one can estimate, assuming an unform $p_{\pi}$ electronic distribution on the central part of the TMTSF molecule, that the amplitude of such an electronic modulation is of about 0.1 electron per molecule. This value is comparable to the amplitude $\left(0.08 \mu_{\mathrm{B}}\right)$ of the SDW. More details can be found in reference [76].

A SDW ground state is exhibited by the Cr. In this material the primary SDW modulation contributes to " $2 k_{\mathrm{F}}$ " satellite reflections, while a CDW modulation, set by magnetoelastic coupling with the SDW, gives rise to satellites at twice this wave vector [29]. The ground state of (TMTSF) ${ }_{2} \mathrm{PF}_{6}$ is different in this respect because both the $2 k_{\mathrm{F}} \mathrm{CDW}$ (seen by X-ray scattering) and the $2 k_{\mathrm{F}} \mathrm{SDW}$ (seen by magnetic measurements) contribute at the primary modulation. Such a mixed $2 k_{\mathrm{F}} \mathrm{CDW}-\mathrm{SDW}$ modulation has been considered long time ago by Overhauser [30]. It can be constructed from the combination of two electronic CDW of opposite spin, taken, in the simplest case, as sine functions:

$$
\begin{array}{r}
\delta \rho_{\uparrow}(x)=\frac{\alpha \bar{\rho}}{2} \cos \left(2 k_{\mathrm{F}} x+\varphi\right) \\
\delta \rho_{\downarrow}(x)=\frac{\alpha \bar{\rho}}{2} \cos \left(2 k_{\mathrm{F}} x+\varphi+\theta\right)
\end{array}
$$

where $\bar{\rho}$ is the average electronic density and $\alpha$ is a number $\leq 1$. A pure CDW corresponds to $\delta \rho_{\uparrow}+\delta \rho_{\downarrow}$, with the two waves in phase $(\theta=0)$. A pure SDW corresponds to $\delta \rho_{\uparrow}-\delta \rho_{\downarrow}$ with the two waves out of phase $(\theta=\pi)$. A mixed CDW-SDW is obtained for an arbitrary phase shift $\theta$ between $\delta \rho_{\uparrow}$ and $\delta \rho_{\downarrow}$. The mechanism by which a mixed $2 k_{F}$ CDW-SDW ground state is stabilized in (TMTSF) ${ }_{2} \mathrm{PF}_{6}$ is not known. It could be related to the presence of a spin orbit coupling which is important in the Se based TMTSF molecule.

Such a mixed charge-spin character of the modulation could be the clue to resolve the discrepancies previously mentionned in the thermodynamics and dynamics of the SDW ground state of (TMTSF) ${ }_{2} \mathrm{PF}_{6}$. The CDW component could be also at the origin of the "phase phonons" observed by IR in the SDW ground state of (TMTSF) $)_{2} \mathrm{SbF}_{6}$ [31a]. The $2 k_{\mathrm{F}} \mathrm{CDW}$ component could be also present in the field induced SDW phases of (TMTSF) ${ }_{2} \mathrm{ClO}_{4}$; some experiments already show a strong interaction of low energy phonons with the field induced modulation $[31 \mathrm{~b}]$.

\subsection{The $4 k_{\mathrm{F}}$ Charge localization}

3.2.1. General Features. - The electrical conductivity of (TMTSF) ${ }_{2} \mathrm{PF}_{6}$ increases when the temperature decreases until the occurrence of the $T_{N}$ metal insulator phase transition $[3,25]$. Such is not the case for (TMTTF $)_{2} \mathrm{PF}_{6}$ where after a broad maximum of conductivity around 
$230 \mathrm{~K}\left(T_{\rho}\right)$ the conductivity decreases upon cooling [32]. This behavior recalls that of Qn$(\mathrm{TCNQ})_{2}$ and related TCNQ salts [1]. In all these salts IR vibronic intensity measurements show that this feature is due to localized electrons on the TMTTF [33] or TCNQ [34] molecules. implying the presence of a gap of charge $\left(2 \Delta_{\rho}\right)$ on the donor or acceptor stacks. whose effect becomes relevant below $T_{\rho} \sim \Delta_{\rho} / \pi$. In Qn-(TCNQ) $)_{2}$ such charge localization effects were initially thought to be entirely caused by disorders in the structure [1]. However X-ray diffuse scattering experiments were able to show that such a gap of charge can be also associated with the formation of a short range $4 k_{\mathrm{F}} \mathrm{CDW}$, leading to a local dimerization of the TCNQ stacks [17]. Quasi-1D $4 k_{F}$ CDW diffuse scattering effects are not detected in (TMTTF) ${ }_{2} \mathrm{PF}_{6}$ and related salts. As the $4 k_{\mathrm{F}}$ wave vector of these last salts already anounts to the reciprocal lattice periodicity $2 \pi / a$, the $4 k_{\mathrm{F}}$ charge localization can be associated to the lattice dimerization previously considered in Section 2.2 .

It was soon recognized that the effects of a gap of charge $2 \Delta_{\rho}$ are relevant in the (TMTTF) ${ }_{2} \mathrm{X}$ salts because the $4 k_{\mathrm{F}}$ umklapp electron-electron interaction term, $g_{3}$, whose value increases with both the Coulomb repulsions and the dimerization gap $\Delta_{\mathrm{D}}$, is strong enough [35]. $\Delta_{\rho}$ was recently calculated, in function of the reduced intraside Coulomb repulsion $U /\left\langle t_{\mathrm{s}}\right\rangle$ and the reduced dimerization $\Delta_{\mathrm{D}} /\left\langle t_{\mathrm{s}}\right\rangle$, in the framework of the $1 \mathrm{D}$ dimerized Hubbard model at $1 / 4$ (or 3/4) band filling [36a]. $\Delta_{\rho}$ is estimated [36b] at about $\left\langle t_{\mathrm{s}}\right\rangle / 50$ in (TMTSF) ${ }_{2} \mathrm{PF}_{6}$ and at about $\left\langle t_{\mathrm{s}}\right\rangle / 4$ in $(\mathrm{TMTTF})_{2} \mathrm{PF}_{6}$.

With respect to (TMTSF) ${ }_{2} \mathrm{PF}_{6}$, the enhanced value of the gap of charge in (TMTTF $)_{2} \mathrm{PF}_{6}$ $\left(\Delta_{\rho} \sim 600 \mathrm{~K}\right)[32,37]$ is due to both the increase of reduced Coulomb interactions $U /\left\langle t_{\mathrm{s}}\right\rangle$ (i.e. decrease of donor polarızability and of transfer integral $\left.\left\langle t_{\mathrm{s}}\right\rangle\right)$ and of the reduced dimerization $\Delta_{\mathrm{D}} /\left\langle t_{\mathrm{s}}\right\rangle$ (see Tab. I) from the TMTSF stack to the TMTTF stack. A larger gap of charge is found in (BCPTTF $)_{2} \mathrm{PF}_{6}\left(\Delta_{\rho} \sim 1000 \mathrm{~K}[13 \mathrm{~d}]\right)$. Because of that this salt exhibits below RT a good decoupling between the charge and spin degrees of freedom. As a consequence the spin susceptibility follows the Bonner and Fisher thermal dependence of the $S=1 / 2$ antiferromagnetic (AF) chain [38]. An enhancement of the lattice dimerization, obtained by incorporating the bulkier anion $\mathrm{X}=\mathrm{Re}_{6} \mathrm{Se}_{5} \mathrm{Cl}_{9}$ in the (TMTTF) $)_{2} \mathrm{X}$ structure, increases by a factor two the gap of charge $\left(\Delta_{\rho} \sim 1300 \mathrm{~K}\right)$. This salt exhibits also to the Bonner and Fisher like dependence of the spin susceptibility with, as expected from the larger value of $\Delta_{\rho}$, an exchange integral $(J \sim 125 \mathrm{~K})[14]$ slightly less than for $(\mathrm{BCPTTF})_{2} \mathrm{PF}_{6}(J \sim 165 \mathrm{~K})[38]$.

In all these salts. where the donors are related by the $P \overline{1}$ inversion symmetry, $\Delta_{D}$ is due to the $4 k_{\mathrm{F}}$ bond dimerization of the stack (.$e \Delta_{\mathrm{D}}^{\mathrm{b}}$ defined by (1)). If the inversion symmetry is lost the molecules become inequivalent and the difference of their site energy gives rise to an additional $4 k_{F}$ contribution $\left(\Delta_{D}^{s}\right)$ at $\Delta_{D}$. These two components add in quadrature. $\Delta_{D}$ thus
becomes:

$$
\Delta_{\mathrm{D}}=\sqrt{\left(\Delta_{\mathrm{D}}^{\mathrm{b}}\right)^{2}+\left(\Delta_{\mathrm{D}}^{\mathrm{s}}\right)^{2}}
$$

The increase of $\Delta_{\mathrm{D}}$ which thus results leads to an enhancement of the gap of charge $2 \Delta_{\rho}$. This enhancement $\left(\Delta_{\rho} \sim 750 \mathrm{~K}\right)$ is observed in the $\left[(\mathrm{TMTSF})_{1-x}(\mathrm{TMTTF})_{x}\right]_{2} \mathrm{ReO}_{4}$ solid solution where, for $x \sim 0.5$, an alternate order between the TMTSF and TMTTF molecules breaks the site equivalence [12]. A similar value of $\Delta_{\rho}(\sim 750 \mathrm{~K})$ is also measured [32,39] below the $(0,1 / 2,1 / 2)$ structural transition of (TMTTF) $)_{2} \mathrm{SCN}$ where at $160 \mathrm{~K}$ the " $4 k_{\mathrm{F}}$ " order of the dipolar SCN anions breaks the inversion symmetry of the TMTTF stack.

The dependence of $\Delta_{\mathrm{D}}$ with the size and shape of the anions certainly provides the explanation of the drastic variations of the electronic properties of the (DMET) $)_{2} \mathrm{X}$ salts isostructural to the Bechgaard salts [5]. With small anions such as $\mathrm{X}=\mathrm{PF}_{6}$ and $\mathrm{AsF}_{6}$ the $\mathrm{DMET}$ salts, which are already semiconducting at RT, bear some resemblance with the "large $\Delta_{\rho}$ " (TMTTF) $2 \mathrm{X}$ salts. With linear anions such as $\mathrm{X}=\mathrm{AuCl}_{2}, \mathrm{AuI}_{2}$ and $\mathrm{Au}(\mathrm{CN})_{2}$ the DMET salts, which 


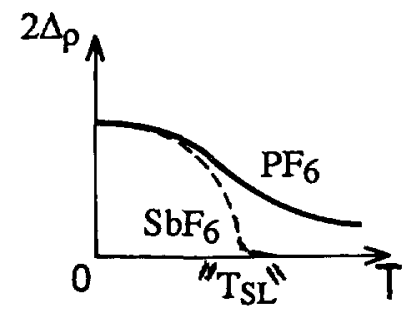

Fig. 6. - Schematical representation of the thermal dependence of the $4 k_{\mathrm{F}}$ gap of charge $\left(2 \Delta_{\rho}\right) \mathrm{m}$ $(\mathrm{TMTTF})_{2} \mathrm{PF}_{6}$ and $(\mathrm{TMTTF})_{2} \mathrm{SbF}_{6}$.

exhibit a metallic behavior down to low temperature, bear some resemblance with the "small $\Delta_{\rho} "$ (TMTSF $)_{2} \mathrm{X}$ salts. As in the Bechgaard salts, these last compounds undergo a superconducting transition, the former one at ambient pressure and the latter two under pressure.

3.2.2. The "Structureless" Transition of (TMTTF) $)_{2} \mathrm{SbF}_{6}$ and $\mathrm{ReO}_{4}$. - In (TMTTF) ${ }_{2} \mathrm{X}$ with small anions such as $\mathrm{X}=\mathrm{PF}_{6}, \mathrm{ClO}_{4}, \mathrm{BF}_{4}$ the electrical conductivity exhibits a smooth thermal dependence with a broad maximum around $T_{\rho} \sim 220 \mathrm{~K}$ [32]. This behavior is due to the formation of a gap of charge $2 \Delta_{\rho}$ whose amplitude gently increases on cooling [33] (Fig. 6). Such a feature can be ascribed to the slight increase of the $4 k_{\mathrm{F}} \mathrm{CDW}$ response of the organic stack to the bond dimerization potential $\Delta_{\mathrm{D}}^{\mathrm{b}}$ previously considered.

In salts with larger anions such as $\mathrm{X}=\mathrm{SbF}_{6}$ and $\mathrm{ReO}_{4}$ the electrical conductivity exhibits a much stronger thermal dependence with a sharp anomaly in its derivative at $154 \mathrm{~K}$ [37] and $225 \mathrm{~K}$ [40] respectively ( $T_{\mathrm{SL}}$ below), which suggests the occurrence of a phase transitions. At $T_{\mathrm{SL}}$ there is also a divergent like behavior of the dielectric constant [39] and a break in the thermal dependence of the thermopower [40]. Conductivity and dielectric measurements suggest the rapid growth of a gap of charge $2 \Delta_{\rho}$ below $T_{\mathrm{SL}}$, as schematically 1llustrated Figure 6. However various X-ray investigations of $(\mathrm{TMTTF})_{2} \mathrm{SbF}_{6}[37,42]$ and $\mathrm{ReO}_{4}[40,41]$ were unable to reveal the formation of additional superlattice reflections or to detect a significant variation of intensity of the main Bragg reflections at $T_{\mathrm{SL}}$. Furthermore the refinement of the structure of the $\mathrm{SbF}_{6}$ salt on each side of $T_{\mathrm{SL}}$ was unable to reveal important structural modifications [42]. Such negative structural results tend to accredit the hypothesis that the " $T_{\mathrm{SL}}$ phase transition' has a purely electronic orıgin. Such a "transition" could be driven by the "divergence" of the $4 k_{\mathrm{F}}$ CDW electronic response function of the organic stack. However the observation of a critical behavior requires the nearly absence of the dimerization gap $2 \Delta_{\mathrm{D}}^{\mathrm{b}}$, which is the field conjugated to the $4 k_{\mathrm{F}} \mathrm{CDW}$ order parameter.

This interpretation implies that the magnitude of $\Delta_{\mathrm{D}}^{\mathrm{b}}$ must decrease when the size of the anion increases. Among the two contributions at $\Delta_{\mathrm{D}}^{\mathrm{b}}$, explicitly given by $(1), V_{1}-I_{2}$, which mostly corresponds to the $4 k_{\mathrm{F}}$ bond potential due to the anion sublattice, could vary with the anion size and nearly compensates $t_{\mathrm{s} 1}-t_{\mathrm{s} 2}$ in the $\mathrm{SbF}_{6}$ salts. This $4 k_{\mathrm{F}}$ bond potential. which is proportional to the $4 k_{\mathrm{F}}$ Fourier component of the anion1c charge distribution, could decrease when, for larger anions, the charge will be more uniformly distributed along the stack direction. A similar decrease of this potential is expected for the long linear anions in (DMET) $2 \mathrm{X}$.

\subsection{ThE “ $2 k_{\mathrm{F}}$ ” INSTABILITIES}

3.3.1. General Aspects. - The $2 k_{\mathrm{F}}$ instability of the $2: 1$ cation radical salts must be discussed with respect to the importance of the $4 k_{\mathrm{F}}$ charge localization phenomena previously considered in Section 3.2. In absence of a gap of charge the $2 k_{\mathrm{F}}$ instability is that exhibited by a $1 / 4$ (or 
$3 / 4)$ filled quasi-1D metal. This is the case of $(\mathrm{FA})_{2} \mathrm{PF}_{6}$ which exhibits a regular $2 k_{\mathrm{F}} \mathrm{CDW}$ Peierls transition at $180 \mathrm{~K}$ [43]. Although $(\mathrm{FA})_{2} \mathrm{PF}_{6}$ has a larger structural dimerization than (TMTSF) ${ }_{2} \mathrm{PF}_{6}$, a $4 k_{\mathrm{F}} \mathrm{CDW}$ is certainly not formed because of the efficient screening of Coulomb interactions due to the strong polarizability of the fluorenthene (FA) molecule composed of four rings.

When there is a sizeable $4 k_{\mathrm{F}}$ gap, $2 \Delta_{\rho}$, which freezes the charge degrees of freedom (one charge localized per dimer), two kinds of " $2 k_{\mathrm{F}}$ " ground states, involving the spin degrees of freedom, are observed:

- the antiferromagnetic (AF) order of the spins: case of (TMTTF $)_{2} \mathrm{Br}\left(T_{\mathrm{N}}=13 \mathrm{~K}\right)$ and $(\mathrm{t}-\mathrm{TTF})_{2} \mathrm{Br}\left(T_{\mathrm{N}}=33 \mathrm{~K}\right)$;

- the pairing of the spins in a singulet $(S=0)$ non-magnetic ground state via a tetramerization of the stack (i.e spin-Peierls (SP) transition): case of (TMTTF) ${ }_{2} \mathrm{PF}_{6}\left(T_{\text {sp }}=15 \mathrm{~K}\right.$ ) and $(\mathrm{BCPTTF})_{2} \mathrm{PF}_{6}\left(T_{\mathrm{sp}}=36 \mathrm{~K}\right)$.

In presence of a sizeable $4 k_{F}$ bond localization (case of the TMTTF and BCPTTF salts) only the $2 k_{\mathrm{F}}$ bond CDW response survives [44]. This response, strongly enhanced below $T_{\rho}$, drives the SP lattice softening when coupled to the phonons (see next part). However in presence of an additional $4 k_{\mathrm{F}}$ site potential (i.e $\Delta_{\mathrm{D}}^{\mathrm{s}}$ ) the charge distribution will no longer be "homopolar" within the unit cell. The ionicity of the charge distribution will diminish the resonating character of the bonds and thus wll decrease the singularity of the $2 k_{F}$ bond response [12]. This remark, which explains the physical properties of the ordered solid solution $\left[(\mathrm{TMTSF})_{1-x}(\mathrm{TMTTF})_{x}\right]_{2} \mathrm{X}$ for $x \sim 0.5$, however requires further theoretical investigations.

By continuity the AF order evolves towards a $2 k_{\mathrm{F}} \mathrm{SDW}$ and the SP distortion evolves towards a $2 k_{F}$ CDW when $\Delta_{\rho}$ decreases. Theory predicts $[35,45]$ that when $\Delta_{\rho}$ decreases the dominant instability changes from SP to $2 k_{\mathrm{F}} \mathrm{SDW}$ in agreement with the pressure behavior of $(\mathrm{TMTSF})_{2} \mathrm{PF}_{6}$ and of $(\mathrm{TMTTF})_{2} \mathrm{PF}_{6}$ [18]. We have however seen (Sect. 3.1) that in $(\mathrm{TMTSF})_{2} \mathrm{PF}_{6} 1 \mathrm{D} 2 k_{\mathrm{F}}$ structural CDW fluctuations survive between $20 \mathrm{~K}$ and $150 \mathrm{~K}$ and that the $2 k_{\mathrm{F}}$ ground state is more complex than a simple SDW modulation. Nearly critical $1 \mathrm{D}$ " $2 k_{\mathrm{F}}$ " structural fluctuations are exhibited by salts with a modest charge localization $\left(T_{\rho} \sim 100 \mathrm{~K}\right)$ :

- in (TMDTDSF) ${ }_{2} \mathrm{PF}_{6} 2 k_{\mathrm{F}}$ fluctuations of intermediate CDW-SP character grow from $150 \mathrm{~K}$ until about $20 \mathrm{~K}$ [11]. Below this temperature, when the $2 k_{\mathrm{F}} \mathrm{SDW}$ fluctuations begin to diverge [46], the intensity of the SP fluctuations drops by a factor two;

- in (TMTTF $)_{2} \mathrm{Br}$ weak SP fluctuations grow critically below $70 \mathrm{~K}$ before dropping to zero in the AF ground state. Furthermore a preliminary investigation [76] reveals the formation below $T_{\mathrm{N}}(=13 \mathrm{~K})$, in the $\mathrm{H}=$ odd integer layers of main Bragg reflections, of superlattice reflections which recall the $\mathbf{q}_{2}$ satellites of (TMTSF) ${ }_{2} \mathrm{PF}_{6}$. However the satellite reflections of $(\mathrm{TMTTF})_{2} \mathrm{Br}$ are more intense and are observed at larger scattering wave vectors than the $\mathrm{q}_{2}$ satellites of (TMTSF) ${ }_{2} \mathrm{PF}_{6}$.

3.3.2. The Spin Peierls Instability: - Quas1-1D critical SP fluctuations are observed at the reduced " $2 k_{\mathrm{F}}$ " $=\pi / a$ wave vector in $(\mathrm{TMTTF})_{2} \mathrm{PF}_{6}$ [16b] and $\mathrm{AsF}_{6}$ [37] below about $60 \mathrm{~K}$ and $40 \mathrm{~K}$ respectively. As shown Figure 4 the $\mathrm{SP}$ response function of the $\mathrm{PF}_{6}$ salt diverges on cooling down, leading to a structural phase transition at about $T_{\mathrm{sp}}=15 \mathrm{~K}$. Below $T_{\mathrm{sp}}$ satellite reflections are observed at the reduced wave vector $\mathbf{q}_{\mathrm{sp}}=(1 / 2,1 / 2,1 / 2)$. Unfortunately an accurate study of the SP transition of (TMTTF $)_{2} \mathrm{X}$ could not be performed because of the great sensitivity of this ground state to X-ray irradiation damages. Such a study has been recently performed in isostructural (BCPTTF) ${ }_{2} \mathrm{PF}_{6}$ and $\mathrm{AsF}_{6}[38,47]$ salts which are less sensitive to $\mathrm{X}$-ray irradiation.

The charges are more strongly localized in (BCPTTF) $)_{2} \mathrm{X}$ than in (TMTTF) ${ }_{2} \mathrm{X}$ so that the uniform spın susceptibility $\chi_{\mathrm{s}}$ of the BCPTTF salt exhibits above $100 \mathrm{~K}$ the Bonner and Fisher 
(B.F.) thermal dependence expected for a $S=1 / 2 \mathrm{AF}$ chain (Fig. Ta). Below $T_{\mathrm{F}}(\sim 100 \mathrm{~K}$ in the $\mathrm{PF}_{6}$ salt) there is a net reduction of $\chi_{\mathrm{s}}$ from the extrapolated $\mathrm{BF}$ behavior, followed by a rapid drop of $\chi_{\mathrm{s}}$ below $T_{\mathrm{sp}}\left(=36 \mathrm{~K}\right.$ in the $\mathrm{PF}_{6}$ salt $)$. The occurrence of a SP ground state is confirmed by the observation of the $\mathbf{q}_{\mathrm{sp}}=(1 / 2,1 / 2,1 / 2)$ satellite reflections below $T_{\mathrm{sp}}$ [38]. The SP transition of (BCPTTF $)_{2} \mathrm{X}$ is announced by an important regime of pretransitional fluctuations which can be observed up to $100 \mathrm{~K}$ in the $\mathrm{PF}_{6}$ salt. At this temperature the correlation length of the intrachain fluctuations $\left(\xi_{a}\right)$ amounts to the a parameter ( interdimer or interspin separation). Between $50 \mathrm{~K}$ and $100 \mathrm{~K}$ the fluctuations are basically $1 D$ (the interchain correlation length is less than the interchain distance). Figures $T b$ and $c$ give respectively the thermal dependence of the inverse SP response function, $\chi_{\mathrm{sp}}^{-1}$. and of the square of the inverse intrachain correlation length, $\xi_{a}^{-2}$, in the $\mathrm{PF}_{6}$ salt. Both quantities show that the SP fluctuations diverge at $T_{\mathrm{sp}}$, as expected for a $2^{\text {nd }}$ order transition. It is also interesting to remark that the deviation at the B.F. thermal dependence occurs consistently below $T_{\mathrm{F}} \sim 100 \mathrm{~K}$ (Fig. $7 \mathrm{a}$ ) when there is spin parring on a larger distance $\xi_{a}$ than the interspin separation. The influence of such $1 \mathrm{D}$ fluctuations on the thermal behavior of $\gamma_{s}$ and of $\xi_{a}$ has been recently calculated [38b]. The theory accounts quantitatively the experimental data obtained on the $(\mathrm{BCPTTF})_{2} \mathrm{X}$ salts.

\section{The Anion Ordering Transitions}

4.1. General ASPECTS. - We have seen Section 2.3 that non centrosymmetrical anions X incorporated in the cavities delimited by the TMTSF or TMTTF molecules are disordered at $\mathrm{RT}$. In this case the (TMTSF $)_{2} \mathrm{X}$ or $(\mathrm{TMTTF})_{2} \mathrm{X}$ salts formed with these anions undergo a structural transition upon cooling [48]. The critical temperature $\left(T_{\mathrm{AO}}\right)$, the critical wave vector $\left(\mathbf{q}_{\mathrm{AO}}\right)$ and the $1^{\text {st }}$ or $2^{\text {nd }}$ order nature of this transition are summarized by Table II. In first, it is interesting to remark that $T_{\mathrm{AO}}$ is significantly larger that the critical temperature of the various phase transitions driven by the donor stack $\left(T_{\mathrm{sp}} \sim 15 \mathrm{~K}, \mathrm{~T}_{\mathrm{N}} \sim 10 \mathrm{~K}\right.$ and $\left.T_{\mathrm{s}} \sim 1 \mathrm{~K}\right)$.

a)

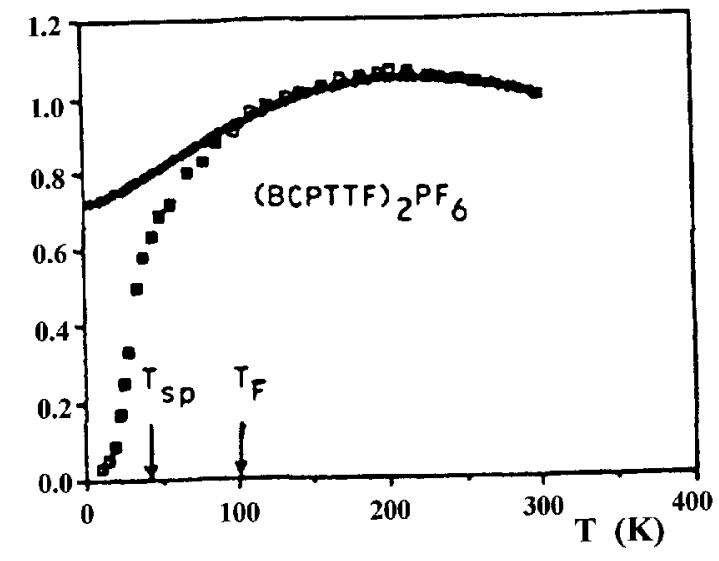

Fig. 7. - a) Thermal dependence of the spin susceptibility, $\chi_{s}$. of (BCPTTF $)_{2} \mathrm{PF}_{6}$. The solid line is the best fit of its high temperature dependence by a Bonner and Fisher behavior (from Ref. [38a]). b) Thermal dependence of the inverse SP response function $\left(\chi_{\mathrm{SP}}^{-1}=T / I\right.$, where $I$ is the $\mathrm{q}_{\mathrm{sp}}$ peak intensity of the X-ray diffuse scattering) (from Ref. [47]). c) Thermal dependence of the half width at half maximum (H.W.H.M.) of the $\mathbf{q}_{\mathrm{sp}}$ X-ray diffuse scattering, $\Delta q_{a}$, and of the square of this quantity corrected by the experimental resolution $\left(\xi_{a}^{-2}\right)$ (from Ref. [47]). 
b)
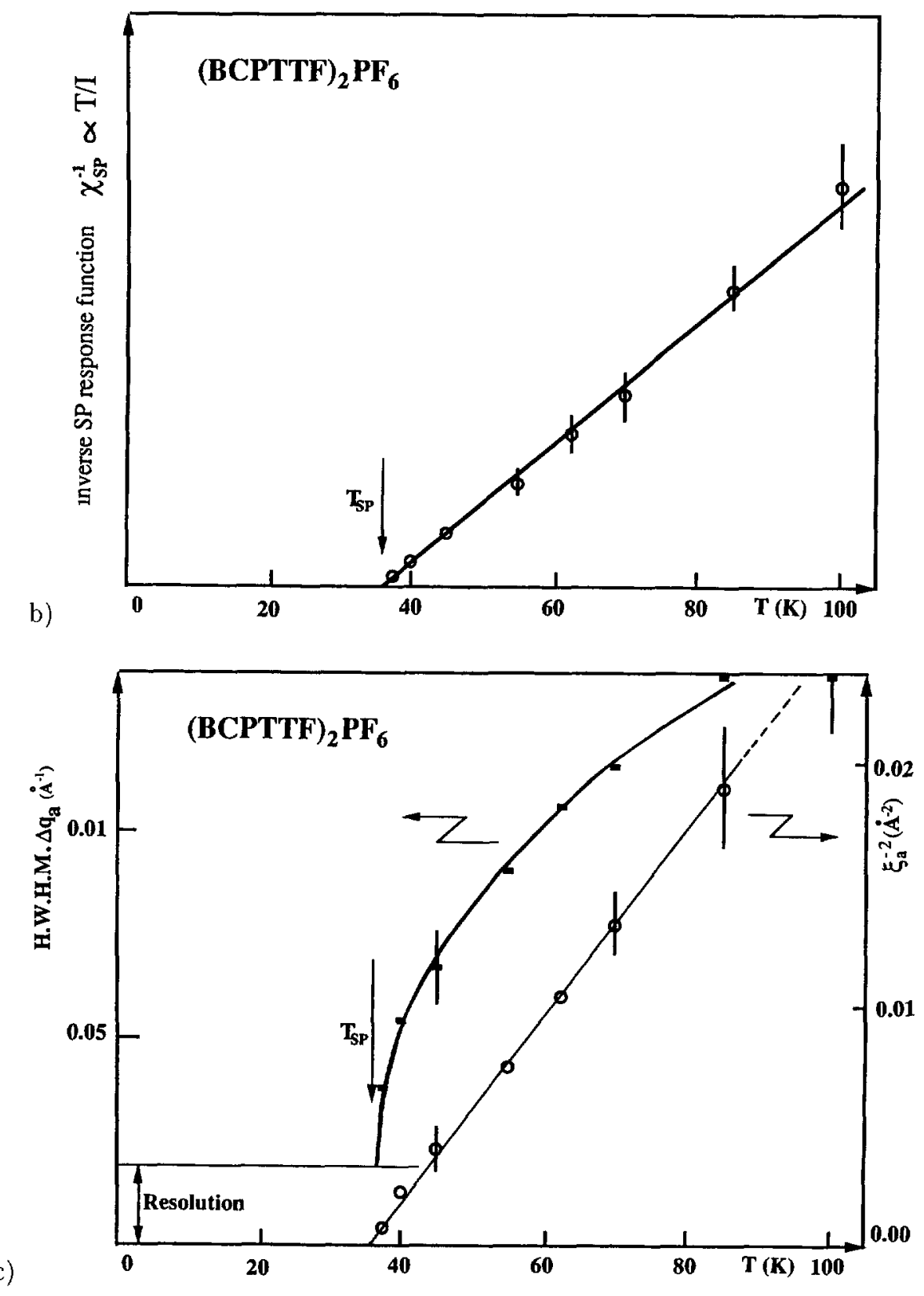

Fig. 7. - (continued)

In the (TMTTF) ${ }_{2} \mathrm{X}$ family $T_{\mathrm{AO}}$ is also, at ambient pressure, lower that the characteristic temperature, $T_{\rho}$, associated to the $4 k_{\mathrm{F}}$ charge localization phenomena.

Structural refinements performed on the (TMTSF) ${ }_{2} \mathrm{ReO}_{4}$ [49], (TMTSF) ${ }_{2} \mathrm{BF}_{4}$ [50], $(\mathrm{TMTSF})_{2} \mathrm{ClO}_{4}$ [51], (TMTSF) ${ }_{2} \mathrm{NO}_{3}$ [13c] and (TMTTF) ${ }_{2} \mathrm{ReO}_{4}$ [48i] salts show that the anlons adopt a well defined orientation below $T_{\mathrm{AO}}$. In most of the cases simple superstructures, characterized by $\mathrm{q}_{\mathrm{AO}}=(1 / 2,1 / 2,1 / 2),(0,1 / 2,1 / 2),(1 / 2,0,0)$ or $(0,1 / 2,0)$, where there is a 
Table II. - Critical temperature $\left(T_{\mathrm{AO}}\right)$, critical wave vector $\left(\mathbf{q}_{\mathrm{AO}}\right)$ and order of the anion ordering phase transition of some (TMTSF) ${ }_{2} X$ and $(T M T T F)_{2} X$ salts (from Ref. [48]). (*) Only a short range order is observed in $(T M T S F)_{2} S C N$ below $T_{\mathrm{AO}}$.

\begin{tabular}{|c|c|c|c|c|c|c|c|c|}
\hline & \multicolumn{4}{|c|}{$(\mathrm{TMTSF})_{2} \mathrm{X}$} & \multicolumn{4}{|c|}{$(\mathrm{TMTTF})_{2} \mathrm{X}$} \\
\hline $\mathrm{X}$ & $T_{\mathrm{AO}}$ & order & $\mathrm{q}_{\mathrm{AO}}$ & Ref. & $T_{\mathrm{AO}}$ & order & qAO & Ref. \\
\hline $\mathrm{ReO}_{4}$ & $\begin{array}{c}176 \mathrm{~K} \\
(1 \mathrm{bar}) \\
240 \mathrm{~K} \\
\text { (17 kbars) }\end{array}$ & $\begin{array}{l}1^{\text {st }} \\
2^{\text {nd }}\end{array}$ & $\begin{array}{c}(1 / 2.1 / 2,1 / 2) \\
(0,1 / 2,1 / 2)\end{array}$ & $\begin{array}{l}\mathrm{a}, \mathrm{j} \\
\mathrm{b}\end{array}$ & $\begin{array}{l}154 \mathrm{~K} \\
(1 \mathrm{bar})\end{array}$ & $\begin{array}{c}1^{\text {st }} \\
\text { (weakly) }\end{array}$ & $(1 / 2,1 / 2,1 / 2)$ & $i, e$ \\
\hline $\mathrm{BF}_{4}$ & $\begin{array}{c}36 \mathrm{~K} \\
(1 \mathrm{bar})\end{array}$ & $1^{\mathrm{st}}$ & $(1 / 2,1 / 2,1 / 2)$ & $\mathrm{c}$ & $\begin{array}{c}40 \mathrm{~K} \\
(1 \mathrm{bar}) \\
\end{array}$ & $1^{\text {st }}$ & $(1 / 2,1 / 2,1 / 2)$ & $\mathrm{d}$ \\
\hline $\mathrm{FSO}_{3}$ & $\begin{array}{l}87.5 \mathrm{~K} \\
(1 \mathrm{bar}) \\
\end{array}$ & $1^{\mathrm{st}}$ & $(1 / 2,1 / 2,1 / 2)$ & $\mathrm{d}$ & $\begin{array}{c}58 \mathrm{~K} \\
(1 \mathrm{bar}) \\
\end{array}$ & $1^{\text {st }}$ & $?$ & \\
\hline $\mathrm{PF}_{2} \mathrm{O}_{2}$ & $\begin{array}{c}136.3 \mathrm{~K} \\
(1 \mathrm{bar}) \\
135.3 \mathrm{~K} \\
(1 \mathrm{bar})\end{array}$ & $\begin{array}{l}2^{\text {nd }} \\
1^{\text {st }}\end{array}$ & $\begin{array}{c}(1 / 2, \pm 1 / 4,1 / 2) \\
(1 / 2,1 / 2,1 / 2)\end{array}$ & $e, f$ & & & & \\
\hline $\mathrm{ClO}_{4}$ & $\begin{array}{c}24 \mathrm{~K} \\
(1 \mathrm{bar})\end{array}$ & $2^{\text {nd }}$ & $(0,1 / 2,0)$ & g & $\begin{array}{c}70 \mathrm{~K} \\
(1 \mathrm{bar})\end{array}$ & $1^{\text {st }}$ & $(1 / 2.1 / 2,1 / 2)$ & j \\
\hline $\mathrm{NO}_{3}$ & $\begin{array}{c}41 \mathrm{~K} \\
(1 \mathrm{bar})\end{array}$ & $2^{\text {nd }}$ & $(1 / 2,0,0)$ & $a, j$ & $\begin{array}{c}50 \mathrm{~K} \\
(1 \mathrm{bar})\end{array}$ & $2^{\text {nd }}$ & $(1 / 2,0,0)$ & $\bar{d}$ \\
\hline $\mathrm{SCN}$ & $\begin{array}{c}90 \mathrm{~K} \\
(1 \mathrm{bar})\end{array}$ & $\begin{array}{l}\text { see } \\
(*)\end{array}$ & $(0.48, \overline{0.35}, 0.1)$ & $\mathrm{h}$ & $\begin{array}{l}160 \mathrm{~K} \\
(1 \mathrm{bar})\end{array}$ & $2^{\text {nd }}$ & $(0,1 / 2,1 / 2)$ & $\mathrm{k}$ \\
\hline
\end{tabular}

staggered orientation of the anions along the [111], [011], [100] or [010] crystallographic directions respectively, are stabilized below $T_{\mathrm{AO}}$. Two exceptions are (TMTSF) ${ }_{2} \mathrm{PF}_{2} \mathrm{O}_{2}$, formung a superstructure characterized by the critical wave vector $(1 / 2, \pm 1 / 4,0)$ in a very narrow temperature range, and (TMTSF) ${ }_{2} \mathrm{SCN}$ which exhibits a short range incommensurate modulation with a critical wave vector close to $(1 / 2, \overline{1 / 3}, 0)$. Structural refinements show that the ordering of the anion is also accompanied by its shift $(\sim 0.1 \AA)$ from the center of the cavity delimited by the organic molecules. In the case of the $(1 / 2,1 / 2,1 / 2)$ superstructure a sizeable tetramer1zation (molecular displacements of about $0.1 \AA$ ) of the organic stacks accompanies the anion ordering (AO). The organic stack is nearly not perturbated (displacements less than $10^{-2} \AA$ ) at the $(0,1 / 2,0) \mathrm{AO}$ of $(\mathrm{TMTSF})_{2} \mathrm{ClO}_{4}$ [51] and at the $(1 / 2,0,0) \mathrm{AO}$ of (TMTSF) ${ }_{2} \mathrm{NO}_{3}[13 \mathrm{c}]$.

The AO transition leads to the orientation and the displacement of charged entities inside the organic cavities and for $\mathbf{q}_{\mathrm{AO}}=(1 / 2,1 / 2,1 / 2)$ to the deformation of these cavities. In addition the $\mathrm{AO}$ is generally accompanied by an elastic deformation of the $\mathrm{P} \overline{1}$ triclinic lattice. Important variations of the lattice parameters have been observed below $T_{\mathrm{AO}}$ in the case of the $(1 / 2,1 / 2,1 / 2) \mathrm{AO}$ of $(\mathrm{TMTSF})_{2} \mathrm{ReO}_{4}$ [52] and of $\mathrm{PF}_{2} \mathrm{O}_{2}$ [48f]. Less important lattice deformations have been also detected at the $(0,1 / 2,0) \mathrm{AO}$ of (TMTSF) ${ }_{2} \mathrm{ClO}_{4}$ [53]. Table I shows that all the $(1 / 2,1 / 2,1 / 2) \mathrm{AO}$ are stabilized after a $1^{\text {st }}$ order phase transition while the other $\mathrm{q}_{\mathrm{AO}} \mathrm{AO}$ are achieved by a $2^{\text {nd }}$ order phase transition.

The non centrosymmetrical anions which are located in centrosymmetrical cavities have to choose their orientation between one of the two symmetry related orientations, noted I and II in 
Figure 2a. The local potential experienced by each anion from its surrounding is thus composed of two symmetrical wells. as schematically shown Figure $2 \mathrm{~b}$. In order to change 1ts orientation the anion has to overcome a barrier potential $V_{0}$. If $V_{0}$ is much larger than $k_{\mathrm{B}} T_{\mathrm{AO}}$ the kinetic effects associated to individual reorientations will influence the occurrence of the AO. This is the case of (TMTSF) ${ }_{2} \mathrm{ClO}_{4}$ where $V_{0} / k_{\mathrm{B}}$ (estimated at $240 \mathrm{~K}$ from NMR measurements) is one order of magnitude larger than $T_{\mathrm{AO}}(24 \mathrm{~K})$. In such a case the fraction of ordered anions depends crucially on the cooling rate of the sample near $T_{\mathrm{AO}}$ [54]. It is even possible that the increase of $V_{0}$ under pressure could prevent the establishment of the $\mathrm{AO}$ transition of (TMTSF) ${ }_{2} \mathrm{ClO}_{4}$ above 4 kbars, as recently suggested by electronic measurements [55]. Low frequency (in the $\mathrm{Hz}$ range) dependent effects have been also observed [56] on the thermodynamics of the $\mathrm{AO}$ transition of (TMTSF) ${ }_{2} \mathrm{BF}_{4}$. which $T_{\mathrm{AO}}$ is of $36 \mathrm{~K}$.

4.2. Mechanism of the AO TRAnsition. - The microscopic interactions leading to the AO transitions are not accurately known. The main coupling energy between the anions is of Coulomb origin. The observation of quasi-isotropic 3D pretransitional fluctuations [48a,j] on a sizeable temperature $(T)$ range above $T_{\mathrm{AO}}$ can be understood by the presence of such Coulomb forces. In many salts the observation of $3 \mathrm{D}$ fluctuations and the occurrence of the $\mathrm{AO}$ transition in the $T$ range where the $2 k_{\mathrm{F}} \mathrm{CDW}$ or SP fluctuations are not clearly developped (see Fig. 4 for the $\mathrm{PF}_{6}$ salts) mean that the $1 \mathrm{D}$ electron gas located on the organic stacks does not provide the driving force of the $\mathrm{AO}$ transition. However its underlying $2 k_{\mathrm{F}}$ instability certainly help to stabilize the $(1 / 2,1 / 2,1 / 2)$ superstructure by the gain of electronic energy due to the opening of a $2 k_{\mathrm{F}}$ gap in the charge and/or spin degrees of freedom. In presence of these couplings the AO transition occurs upon cooling for entropy reasons. In the case of the TMTSF [57] and TMTTF [32] salts of $\mathrm{ClO}_{4}$ the entropy of transition per formulae unit, $\Delta S$, is close to $k_{\mathrm{B}} \ln 2$, a value expected for the $\mathrm{AO}$ between two equivalent orientations. $\Delta S$ is however less than this ideal value in the (TMTSF) ${ }_{2} \mathrm{BF}_{4}$ and $\mathrm{ReO}_{4}$ salts [56].

In salts containing tetrahedral anions such as $\mathrm{ReO}_{4}$ and $\mathrm{ClO}_{4}$ the superlattice periodicity stabilized by the AO seems to depend upon a subtle balance between direct Coulomb interactions between anions, which favor an uniform $\left(q_{a}=0\right)$ ordering of tetrahedra in stack direction. and mediated interactions, through the enhanced $2 k_{F}$ polarizability of the organic stack, which favor an alternate $\left(q_{a}=\pi / a \equiv 2 k_{\mathrm{F}}\right)$ ordering in stack direction. At ambient pressure the mediated interactions dominate in salts with anions, such as $\mathrm{ReO}_{4}$, in strong contact with the organic stack and in the TMTTF salts where the SP response function exhibits a low temperature divergence [58]. In that case the $(1 / 2,1 / 2,1 / 2)$ superstructure formation is accompanied by a sizeable stack tetramerization. Direct interactions are dommant in the TMTSF salts where the anions are in weaker contact with the organic stack. This in the case of (TMTSF) ${ }_{2} \mathrm{ClO}_{4}$ which finally stabilizes the $(0,1 / 2,0)$ AO superstructure after a competition with high temperature $(1 / 2,1 / 2,1 / 2) \mathrm{AO}$ fluctuations [59]. The delicate balance between mediated and direct interactions can be reversed under pressure. This is the case of (TMTSF) ${ }_{2} \mathrm{ReO}_{4}$ whose superstructure critical wave vector changes from $(1 / 2,1 / 2,1 / 2)$ to $(0,1 / 2,1 / 2)$ above about 9 kbars [48b].

The $\mathbf{q}_{\mathrm{AO}}$ of the superstructure stabilized below $T_{\mathrm{AO}}$ seems also to depend on the shape of the anion. The $(1 / 2,0,0) \mathrm{AO}$ is only found with the triangular $\mathrm{NO}_{3}$ anion. It could be mainly achieved by direct interactions between the $\mathrm{NO}_{3}$ which display very weak contacts with the organic stack. In (TMTSF) ${ }_{2} \mathrm{NO}_{3}$ the finding below $T_{\mathrm{AO}}$ [13c] of an extremely weak tetramerization of the organıc stack suggests weak mediated interactions. The $(1 / 2, \pm 1 / 4,0)$ order stabilized in a very narrow temperature range in (TMTSF) ${ }_{2} \mathrm{PF}_{2} \mathrm{O}_{2}$ could be due to the dipolar nature of the anion [48f]. 


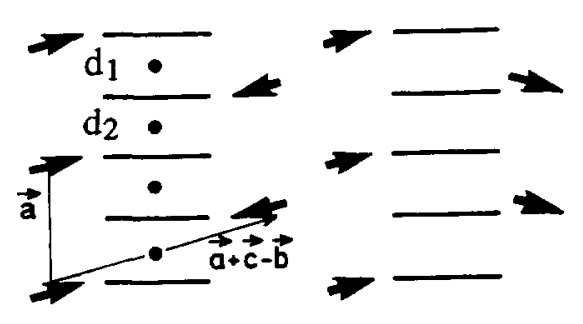

(I)

(II)

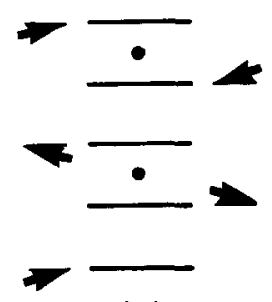

(a)

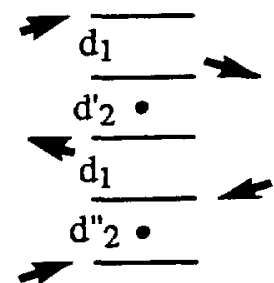

(b)

(III)

Fig. 8. - Schematic representation of organic stacks in the $(\mathbf{a}, \mathbf{c}-\mathbf{b})$ plane for various AO configurations. The arrows symbolize the short contact interaction between the anion and the molecule. $d_{1}$ and $d_{2}$ distances (defined Fig. 1a) are indicated in (I) as well as their modification at the $(1 / 2,1 / 2,1 / 2$ ) $\mathrm{AO}$ in (IIIb). The dots represents the location of the intrastack inversion centers.

4.3. Influence of the AO Transition on the Electronic Properties. - The AO changes the electrostatic potential seen by the $\mathrm{p}_{\pi}$ electrons on the donor stacks and often drives a lattice distortion of the stacks themselves. This transition has thus a considerable influence on the electronic structure of the Bechgaard salts. These effects have been discussed in the literature [60-62] with respect to the change of symmetry of the organic stack induced by the $\mathrm{AO}_{4}$. Figure 8 illustrates three relevant situations based on the inversion symmetry of the short and long contacts ( $\mathrm{I}$ and II in Figs. $1 \mathrm{~b}$ and $2 \mathrm{a}$ ) established by the anions with the organic molecules:

- in $(\mathrm{I})$, corresponding to the $(0,1 / 2,0) \mathrm{AO}$, each stack still experiences a $4 k_{\mathrm{F}}$ bond potential from the anions but, because of the doubling of the $b$ periodicity, there are two inequivalent stacks per unit cell; let say one stack with the anions in short contact (represented here) and one stack with the anions in long contact. As the amonic potential is no longer the same on the molecular sites of each kind of stack, the $1 \mathrm{D}$ band structure of these two kinds of stack is slightly splitted by $2 \Delta_{\mathrm{AO}}$ [61]. The Fermi wave vector thus slightly shifts from each side of $k_{\mathrm{F}}^{0}=\pi / 2 a$ by:

$$
\delta k_{\mathrm{F}}=k_{\mathrm{F}}-k_{\mathrm{F}}^{0}= \pm \hbar v_{\mathrm{F}} \Delta_{\mathrm{AO}}
$$

- in (II), corresponding to the $(0.1 / 2,1 / 2) \mathrm{AO}$, the anions alternatively establish long and short contacts with the organic molecules along the stack so that its inversion symmetry is lost. A $4 k_{\mathrm{F}}$ potential of site is thus created by this pecular $\mathrm{AO}$ periodicity. It adds in quadrature with the $4 k_{\mathrm{F}}$ bond potential (see (4)).

- in (III) the stacks are tetramerized. (a) and (b) differ by the placement of the inversion centers with respect to the anionic sublattice. The configuration (b), corresponding to the $(1 / 2,1 / 2,1 / 2)$ or $(1 / 2,0,0) \mathrm{AO}$, is realized in most of the Bechgaard salts. The $(1 / 2,1 / 4,0) \mathrm{AO}$ of (TMTSF $)_{2} \mathrm{PF}_{2} \mathrm{O}_{2}$ could be described by alternate $(1 / 2,1 / 2,0)$ and $(1 / 2,0,0)$ AO sequences which mixe the (a) and (b) configurations. For the configuration (b) the tetramerization basically consists in a dimerization of the chain of $d_{1}$ dimers. which forms long $\left(d_{2}^{\prime}\right)$ and short $\left(d_{2}^{\prime \prime}\right)$ interdimer distances. In this respect the $(1 / 2,1 / 2,1 / 2)$ AO transition leads to a considerable deformation of the organic stacks with for:

- $(\text { TMTSF })_{2} \operatorname{ReO}_{4}: d_{1} \sim d_{2}^{\prime} \sim 3.6 \AA$ and $d_{2}^{\prime \prime} \sim 3.5 \AA[49]$

- $(\mathrm{TMTSF})_{2} \mathrm{BF}_{4}: d_{1} \sim 3.54 \AA, d_{2}^{\prime} \sim 3.56 \AA$ and $d_{2}^{\prime \prime} \sim 3.5 \AA[50]$

- $(\mathrm{TMTTF})_{2} \mathrm{ReO}_{4}: d_{1} \sim 3.45 \AA, d_{2}^{\prime} \sim 3.6 \AA$ and $d_{2}^{\prime \prime} \sim 3.48 \AA$ [48i] 
In consequence the energy gap, $\sim 0.2 \mathrm{eV}[9]$, opened below $T_{\mathrm{AO}}$ in the (TMTSF) ${ }_{2} \mathrm{ReO}_{4}$ and $\mathrm{BF}_{4}$ salts is comparable to $\left\langle t_{\mathrm{s}}\right\rangle$ or to the Fermi energy $\left(E_{\mathrm{F}}\right)$ of these salts. This means that strong coupling theories are relevant to describe these AO phase transitions. In particular the key role of the anionic potential to drive the metal-insulator phase transition, associated to the $(1 / 2.1 / 2,1 / 2)$ structural transformation, has been already pointed out in the literature [63].

The $(1 / 2,0,0) \mathrm{AO}$ transition of (TMTSF $)_{2} \mathrm{NO}_{3}$ although stabilizating the $2 k_{\mathrm{F}}$ periodicity is not accompanied by a metal-insulator phase transition. The semi-metallic behavior observed below $T_{\mathrm{AO}}$ is probably due to the warping of the Fermı surface which prevents a complete gap opening. It also requires the presence of a weak $2 k_{\mathrm{F}}$ gap $\left(\Delta_{2 k_{\mathrm{F}}}<t_{\perp}\right)$ due to the weak stack tetramerization and the weak change of anionic potential. With a Fermi surface weakly perturbated by the AO, and still keeping its good nesting conditions, a SDW ground state is stabilized below $T_{\mathrm{N}}=8 \mathrm{~K}$, at atmospheric pressure, in (TMTSF) ${ }_{2} \mathrm{NO}_{3}$.

The $(0,1 / 2,1 / 2)$ AO leads to phases which electronic nature differs in the TMTTF and TMTSF salts. In (TMTTF) $2 \mathrm{SCN}$ (where $\Delta_{\rho}$ is relevant below $T_{\rho} \sim 250 \mathrm{~K}$ ) the $\mathbf{q}_{\mathrm{AO}}$ order increases the charge localization phenomena [39] (as the charge and spin degrees of freedom are already decoupled, no change of spin susceptibility is observed at $T_{\mathrm{AO}}=160 \mathrm{~K}$ when the conductivity abruptly decreases [48]). In pressurized (TMTSF) ${ }_{2} \mathrm{ReO}_{4}$, on the other hand, the $(0,1 / 2,1 / 2) \mathrm{AO}$ leads to a better conducting state [64], showing that the $\mathrm{g}_{3}$ unklapp scattering
effects are not relevant, at least above $10 \mathrm{kbars}$.

The $(0.1 / 2,0) \mathrm{AO}$ of (TMTSF $)_{2} \mathrm{ClO}_{4}$ has more subtle effects. In relaxed samples the salt remains metallic down to low temperature and becomes a superconductor. In quenched samples the salt undergoes, at $T_{\mathrm{N}} \sim 4.5-6 \mathrm{~K}$, a SDW transition, as for (TMTSF) ${ }_{2} \mathrm{PF}_{6}$ or pressurized $(\mathrm{TMTSF})_{2} \mathrm{ReO}_{4}$. The absence of a SDW transition is generally attributed to bad nesting conditions of the Fermı surface. We think that this is especially the case of relaxed (TMTSF $)_{2} \mathrm{ClO}_{4}$ because of the splitting of the Fermi wave vectors due to the $(0,1 / 2,0)$ symmetry of the anionic potential. By contrast pressurized (TMTSF) ${ }_{2} \mathrm{ReO}_{4}$, where such a splitting is not achieved by the $(0,1 / 2,1 / 2) \mathrm{AO}$, stabilizes a SDW ground state [64]. This splitting can be also easily destroyed. For example if there is a $(0,1 / 2,0) \mathrm{AO}$ local order (on $\xi_{a} \sim \Delta Q_{a}^{-1}$ ) and if $\delta k_{\mathrm{F}}$ given by (5) becomes less than $\Delta Q_{a}$, the splitting of the Fermi surface will not be relevant so that a SDW could be stabilized by its nesting. This is observed, when $\Delta Q_{a} \geq\left(\Delta Q_{a}\right)_{c} \sim 10^{-2} \AA^{-1}$, in quenched (TMTSF) ${ }_{2} \mathrm{ClO}_{4}$ [54] for rapid enough cooling rates or in alloys with TMTTF [59] or $\mathrm{ReO}_{4}[66]$ for few percent substituant concentrations. This allows to estimate that in $(\mathrm{TMTSF})_{2} \mathrm{ClO}_{4}$ half the anionic potential amounts to about:

$$
\Delta_{\mathrm{AO}} \sim \hbar v_{\mathrm{F}}\left(\Delta Q_{a}\right)_{\mathrm{c}} \sim E_{\mathrm{F}}\left(\Delta Q_{a}\right)_{\mathrm{c}} \frac{\pi}{2 a} \sim 1 \mathrm{meV}
$$

by taking $E_{\mathrm{F}} \sim 0.25 \mathrm{eV}$ and $a=7 \AA$. Consistently $2 \Delta_{\mathrm{AO}} / k_{\mathrm{B}}$ corresponds to $T_{\mathrm{AO}}=24 \mathrm{~K}$. The splitting of the band structure due to the anionic potential has been also recently considered to explain the pecular field induced SDW phase diagram of (TMTSF) ${ }_{2} \mathrm{ClO}_{4}$ and the reentrance of its metallic phase at high magnetic field [65].

4.4. EFFECT OF THE DisORDER. - In order to study the competition between the varlous instabilities exhibited by the Bechgaard salts solid solutions of donors, [(TMTSF) ${ }_{1-x}$ $\left.(\mathrm{TMTTF})_{x}\right]_{2} \mathrm{X}$, and of anıons. $[\mathrm{TMTS}(\mathrm{T}) \mathrm{F}]_{2} \mathrm{X}_{1-x} \mathrm{Y}_{x}$ have been synthetized as well as $2: 1$ salts based on the S/Se hybrid molecule (TMDTDSF) $)_{2} \mathrm{X}$. In these compounds TMTTF/TMTSF or $\mathrm{X} / \mathrm{Y}$ substitutional disorder or TMDTDSF orientational disorder is introduced in the structure. Incomplete $\mathrm{AO}$, achieved by a rapid cooling of (TMTSF) ${ }_{2} \mathrm{ClO}_{4}$ for example, also introduces disorder in the structure at low temperature. These disorders have a profound influence on the electronic instabilities exhibited by these quasi-1D conductors. 
The influence of the cooling rate on the AO transition of (TMTSF) ${ }_{2} \mathrm{ClO}_{4}$ has been studied in detail in reference [54]. Here let us recall that whatever the cooling rate there is always an $(0,1 / 2,0)$ AO:

- for high speed of cooling (>5 K/min) less than $50 \%$ of the $\mathrm{ClO}_{4}$ are ordered. The ordered $\mathrm{ClO}_{4}$ form domains of well defined size $\left(L_{a} \sim \pi \xi_{a} \sim 300 \AA\right)$ embedded in a matrix of disordered $\mathrm{ClO}_{4}$;

- for low speed of cooling $(<1 \mathrm{~K} / \mathrm{min})$ more than $90 \%$ of the $\mathrm{ClO}_{4}$ are ordered. The ordered $\mathrm{ClO}_{4}$ also form domains. These domains, which are in close contact to each others, present a random distribution of size with however an important number of domains of large size $\left(\bar{L}_{a} \sim 1000 \AA\right)$.

We have explained in Section 4.3 how the size of the ordered domains can tune the nesting properties of the Fermı surface and thus the nature of the ground state of (TMTSF $)_{2} \mathrm{ClO}_{4}$.

Let us now consider the effect of the substitution or of the orientational disorder on the AO transition. Figures 1 and 2 show that in each cavity a non-centrosymmetrical anion can point towards the S[Se] atom of one of the two inversion symmetry related near neighbouring TMTTF [TMTSF] molecules. The orientation of the anion is thus specified by an Ising variable $\eta$ whose value is $+1(-1)$ if the anion points towards the molecule labelled I (II). The AO transition can thus be described by a phenomenological Ising Hamiltonian:

$$
H_{0}=\sum_{\imath, 3} J_{\imath \jmath} \eta_{\imath} \eta_{\jmath}
$$

where $J_{\imath j}$ is the interaction between anions located at sites $\imath$ and $j$.

In solid solution [TMTS(T)F $]_{2} \mathrm{X}_{1-x} \mathrm{Y}_{x}$ where a given site can be occupied either by the anion $\mathrm{X}$ or the anion $\mathrm{Y}$ there is a distribution of $J_{2,}$ interactions. If there $1 \mathrm{~s}$ a random distribution of $\mathrm{X}$ and $\mathrm{Y}$ species, as in the (TMTSF $)_{2}\left(\mathrm{ReO}_{4}\right)_{1-x}\left(\mathrm{ClO}_{4}\right)_{x}$ solid solution $[67,68]$, the AO phase diagram will be described by a random interaction Ising model, as the one used to interpret the spin glasses.

If the organic stacks are composed of orientationaly disordered TMDTDSF molecules or of non ordered TMTTF and TMTSF molecules, the anions experience three kinds of environment corresponding to the S-S, Se-S and Se-Se pairs of atoms towards which they can point (Fig. 9). Among them the Se-S environment breaks locally the inversion symmetry of the cavity (for example the $\mathrm{O}$ atom of $\mathrm{ReO}_{4}$ prefers to establish a short contact with the Se than with the $\mathrm{S}$ ). On the site $i$ this effect can be modeled by a local field $h_{2}$ coupled to the order parameter $\eta_{2}$. The Hamiltonian (7) will thus becomes:

$$
H=H_{0}+\sum_{2} h_{\imath} \eta_{\imath}
$$

In case of a random orientational disorder of TMDTDSF [11] or of a random substitution of TMTSF by the TMTTF [67] there is a random distribution of $h_{2}$. The AO phase diagram will be described by a random field Ising model (plus random interactions if the mediated interaction between anıons depends on the $\mathrm{S}$ or Se nature of the atom in close contact with the anion).

Random interaction effects are very important in the (TMTSF $)_{2}\left(\mathrm{ReO}_{4}\right)_{1-x}\left(\mathrm{ClO}_{4}\right)_{x}$ solid solution because (TMTSF) ${ }_{2} \mathrm{ReO}_{4}$ and (TMTSF) $)_{2} \mathrm{ClO}_{4}$ present $\mathrm{AO}$ transitions with different critical q $\mathrm{qO}_{\mathrm{O}}$ (Tab. II). It is found that the (TMTSF) ${ }_{2} \mathrm{ReO}_{4}(1 / 2,1 / 2,1 / 2)$ long range order (LRO) subsists for $x<0.5$ and that the (TMTSF) ${ }_{2} \mathrm{ClO}_{4}(0,1 / 2,0)$ LRO subsists for $x>0.97$. For $0.5<x<0.97$ both kinds of AO develop competing short range orders (SRO). The basic features of the $(T, x)$ phase diagram of this solid solution can be accounted for by mean field treatments of the Ising model with random interactions $[67,68]$. 

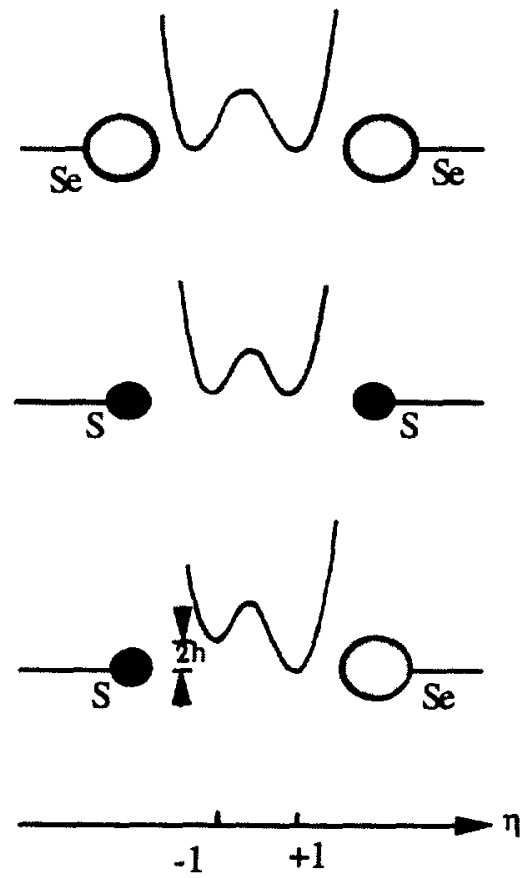

a)

b)

c)

Fig. 9. - Schematic illustration of the lattice potential experienced by an anon in the Se-Se (a), S-S (b) and S-Se (c) environments.

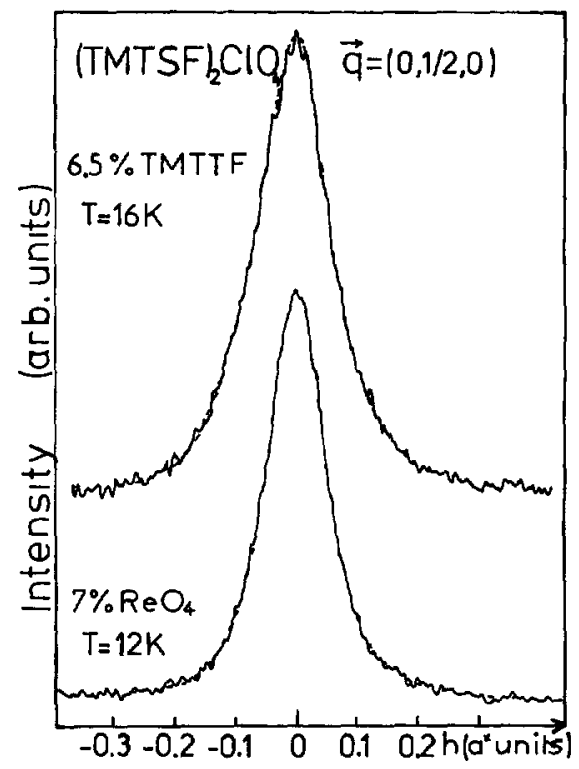

Fig. 10. - Lorentzian square profile along a of the $(0,1 / 2,0)$ superlattice reflections of (TMTSF $)_{2}$ $\mathrm{ClO}_{4}$ substituted with $6.5 \%$ of TMTTF (top) and with $7 \%$ of $\mathrm{ReO}_{4}$ (bottom). 
Random field effects have been considered [11] in (TMDTDSF) ${ }_{2} \mathrm{X}$ salts with $\mathrm{X}=\mathrm{ReO}_{4}$ and $\mathrm{BF}_{4}$. The random interactions are not important in these salts because the (TMTSF) ${ }_{2} \mathrm{X}$ and (TMTTF) $)_{2} \mathrm{X}$ salts develop $(1 / 2,1 / 2,1 / 2) \mathrm{AO}$ with similar $T_{\mathrm{AO}}$ (Tab. II). Random field effects are not relevant in (TMDTDSF) ${ }_{2} \mathrm{ReO}_{4}$ which exhibits a $(1 / 2,1 / 2,1 / 2)$ LRO at a $T_{\mathrm{AO}}(=$ $165 \mathrm{~K}$ ) intermediate between those of (TMTSF) ${ }_{2} \mathrm{ReO}_{4}$ and (TMTTF) $)_{2} \mathrm{ReO}_{4}$. On the other hand random field effects are important in (TMDTDSF) ${ }_{2} \mathrm{BF}_{4}$ which develops a $(1 / 2,1 / 2,1 / 2)$ SRO at lower temperature (" $T_{\mathrm{AO}}$ " $\sim 22 \mathrm{~K}$ ) than the average $T_{\mathrm{AO}}$ of $(\mathrm{TMTSF})_{2} \mathrm{BF}_{4}$ and of $(\mathrm{TMTTF})_{2} \mathrm{BF}_{4}$.

In the SRO case, Figure 10 shows that the profile of the AO superlattice reflections has a lorentzian square shape. Such a profile can be explained by a random distribution of size of ordered domains [69] or by the destruction of the LRO by random fields [70].

\section{Concluding Remarks}

The Bechgaard salts and related compounds exhibit a very rich phase diagram with competing instabilities. Some of these instabilities are caused by the quası-1D $\mathrm{p}_{\pi}$ electron gas. Because of the $1 \mathrm{D}$ character of the electron gas the electron-electron interactions have a magnified importance which can lead, in the sulfur analogues, to a $4 k_{\mathrm{F}} \mathrm{CDW}$ localization and thus to a decoupling between charge and spin degrees of freedom, then finally to SP or AF ground states. In the case of relevant interchain tunneling coupling, which restores the Fermi liquid behavior, the Fermi surface remains open. The nesting of its warped shape leads to $2 k_{\mathrm{F}}$ density wave ground states. When the nesting is suppressed (under pressure or in presence of the $(0,1 / 2,0)$ AO) superconductivity is observed.

The second important feature of the Bechgaard salts is the key influence of the anionic sublattice. In first, such a sublattice controls the degree of dimerization of the organic stacks and thus the nature of its electronic instability. In second, any structural instability or phase transition driven by the anions (such as their ordering) will elther perturb the structural organization of the organic stacks and/or will modify the anionic potential experienced by the $\mathrm{p}_{\pi}$ electrons located on these stacks.

Some of these features will be lost and some of them will remain when the electronic dimensionality of the $\mathrm{p}_{\pi}$ electron gas is increased to two in the BEDTTTF famly of organic salts. The $2 k_{\mathrm{F}}$ instabilities of the electron gas, associated to the nesting properties of its Fermi surface, will be deteriorated by its closing. A " $2 k_{\mathrm{F}}$ " CDW ground state 1 still observed in (BEDTTTF) ${ }_{2} \mathrm{ReO}_{4}$ [71]. However the $1^{\text {st }}$ order nature of this transition is unusual for a CDW-Peierls instability and the interchain interactions are such that its Fermi surface shows both closed and open parts [72]. An incommensurate CDW modulation has been also found in $\beta$-(BEDTTTF $)_{2} \mathrm{~J}_{3}$ [73]. Such a transition is driven by the conformational ordering of the ethylene groups of the BEDTTTF molecule (this modulation is not stabilized in the isostructural $\mathrm{X}=\mathrm{IBr}_{2}$ and $\mathrm{AuI}_{2}$ salts where the ethylene groups are already ordered at $\mathrm{RT}$ ). The ordering of the ethylene groups seems to control the subtle phase diagram exhibited by $\beta$-(BEDTTTF $)_{2} \mathbf{I}_{3}$ [74]. However it has been recently noticed [9] that the experimentally determined intralayer components of the incommensurate modulation seem to achieve a good nesting of the flat parts of its 2D Fermı surface. This nesting effect, which accompanies the ethylene ordering process, could thus help to stabilize the incommensurate modulation. This nesting, which reduces the density of states at the Fermı level could explain why the critical temperature $T_{\mathrm{s}}$ of the supraconducting ground state is depressed in the modulated state of $\beta$-(BEDTTTF $)_{3} I_{3}[5]$.

Very important structural aspects of the (BEDTTTF $)_{2} \mathrm{X}$ salts are conditioned by the presence of hydrogen bond linkages between the anıons $X$ and the layers of BEDTTTF donors [6]. 
Thus any deformation of the anionic sublattice will induce a deformation of the BEDTTTF sublattice, which could have important consequences for the electronic interactions in the conducting layers. In many BEDTTTF salts structural transitions, generally towards simple superstructures, have been observed. This is for example the case of the $12 \mathrm{~K}$ organic superconductor $\kappa$-(BEDTTTF $)_{2} \mathrm{Cu}[\mathrm{N}(\mathrm{CN})] \mathrm{Br}$ which doubles its $c$ lattice periodicity below $200 \mathrm{~K}$ [75]. The driving force of such a phase transition is not known, but the instability could be caused by some constraints having their origin in the anionic sublattice. Further work is necessary in order to characterize the structural transitions exhibited by the BEDTTTF salts and to understand their influence on the electronic properties.

\section{Acknowledgments}

This paper is written in memory of Prof.I.F. Schegolev who has been a pioneer and has always kept a front position in the field of organic conductors. It includes structural results which have been obtained in collaboration with V. Ilakovac, Q. Liu and R. Moret on samples prepared by P. Batail, K. Bechgaard and J.M. Fabre. Some of the new results presented here have benefited from useful discussions with C. Bourbonnais, S. Brazovskii, C. Coulon, L.P. Gor'kov, F. Mila and S. Tomic.

\section{References}

[1] Schegolev I.F., Phys. Stat. Soludi (a) 12 (1972) 9.

[2] Highly Conducting One Dimensional Solids, J.T. Devreese, R.P. Evrard and V.E. Van Doren Eds. (Plenum Press, 1979).

[3] Jerome D. and Schulz H.J., Adv. Phys. 31 (1982) 299.

[4] Highly Conducting Quasi One Dimensional Organic Crystals, E.M. Conwell Ed.. Semıconductors and Semimetals, Vol.27 (Academic Press, 1988).

[5] Ishiguro T. and Yamaji K., Organic Superconductors. Springer Serıes in Solvd State Sciences, Vol. 88 (Springer Verlag, 1990).

[6] Williams J.M., Ferraro J.R., Thorn R.J., Carlson K.D., Geiser U., Wang H.M., Kinn A.M. and Whangbo M.H., Organic Superconductors (Prentice Hall. 1992).

[7] Brossard L., Canadell E., Ravy S., Pouget J.P., Legros J.P. and Valade L., Fizika 21 suppl. 3 (1989) 15.

[8] For two recent short reviews see: Rosseinsky M.J., J. Mater. Chem. 5 (1995) 1497; Tanlgaki K. and Prassides K., J. Mater. Chem. 5 (1995) 1515.

[9] Pouget J.P., Mol. Cryst. Liq. Cryst. 230 (1993) 101.

[10] Thorup N.. Rindof G., Soling H. and Bechgaard K.. Acta Cryst. B37 (1981) 1236.

[11] Liu Q., Ravy S., Pouget J.P., Johannsen I. and Bechgaard K., J. Phys. I France 3 (1993) 803 and 3 (1993) 821.

[12] Ilakovac V., Ravy S., Pouget J.P., Lenoir C., Boubekeur K., Batail P., Dolanski Babic S., Biskup N., Korin-Hamzıc B., Tomıc S. and Bourbonnais C., Phys. Rev. B50 (1994) 7136 , and Synth Metals 70 (1995) 753.

[13] (a) Ducasse L., Abderrabba M., Hoarau J., Pesquer M., Gallons B. and Gaultier J., J. Phys. C19 (1986) 3805; (b) Ducasse L., Abderrabba M., Gallois B. and Chasseau D.. Synth. Metals 19 (1987) 327; (c) Hebrard-Brachetti S., Thesis (Bordeaux, 1996); (d) Ducasse L., 
Coulon C., Chasseau D., Yagbasan R., Fabre J.M. and Gouasmia A.K., Synth. Met. 27 (1988) B 543.

[14] Batail P., Ouahab L., Penicaud A., Lenoir C. and Perrin A., C.R. Acad. Sci. Paris Sér. II 304 (1987) 1111. Renault A., Pouget J.P., Parkin S.S.P., Torrance J.B., Ouahab L. and Batail P., Mol. Cryst. Liq. Cryst. 161 (1988) 329.

[15] Danner G.M., Kang W. and Chaikin P.M., Phys. Rev. Lett. 72 (1994) 3714.

[16] a) Ribault M., Pouget J.P.. Jerome D. and Bechgaard K., J. Phys. Lett. France 41 (1980) L607; b) Pouget J.P., Moret R., Comes R., Bechgaard K., Fabre J.M. and Giral L., Mol. Cryst. Liq. Cryst. 79 (1982) 129.

[17] Pouget J.P., Chem. Scr. 17 (1981) 85.

[18] Wzietek P., Creuzet F., Bourbonnais C., Jerome D., Bechgaard K. and Batail P., J. Phys. I France 3 (1993) 171 and earlier references there in.

[19] Mortensen K., Tomkiewicz Y., Schultz T.D. and Engler E.M., Phys. Rev. Lett. 46 (1981) 1234.

[20] Torrance J.B., Pedersen H.J. and Bechgaard K., Phys. Rev. Lett. 49 (1982) 881.

[21] Le L.P. et al., Europhys. Lett. 15 (1991) 547 and Phys. Rev. B48 (1993) 7284.

[22] Delrieu J.M., Roger M.,Toffano Z., Moradpour A. and Bechgaard K., J. Phys. France 47 (1986) 839; Takahashi T., Maniwa Y., Kawamura H. and Saito G., J. Phys. Soc. Jpn 55 (1986) 1364.

[23] For a recent review see G. Gruner, Rev. Mod. Phys. 66 (1994) 1.

[24] Slater J.C., Phys. Rev. 82 (1951) 538.

[25] Tomic S., Cooper J.R., Kang W., Jerome D. and Maki K., J. Phys. I. France 1 (1991) 1603.

[26] Odin J., Lasjaunıs J.C., Biljakovic K., Monceau P. and Bechgaard K., Solid State Commun. 91 (1994) 523.

[27] It is shown reference [25] that $T_{\mathrm{N}}$ increases by $1 \mathrm{~K}$ for $4 \times 10^{-3}$ mole\% defect. During the time required to take an X-ray pattern, such as those shown Figure 5 , about $10^{-3}$ mole\% defect are created.

[28] Blume M., J. Appl. Phys. 57 (1985) 3615.

[29] Hill J.P., Helgesen G. and Gibbs D., Phys. Rev. B. 51 (1995) 10336 and earlier references there in.

[30] Overhauser A.W., Phys. Rev. 167 (1968) 691.

[31] a) Ng H.K., Timusk T. and Bechgaard K., Phys. Rev. B30 (1984) 5842; b) Janssen T.J.B.M. et al., Phys. Rev. B46 (1992) 8663.

[32] Coulon C., Delhaes P., Flandrois S., Lagnier R., Bonjour E. and Fabre J.M., J. Phys. France 43 (1982) 1059.

[33] Bozio R., Meneghetti M. and Pecille C., J. Chem. Phys. 76 (1982) 5785.

[34] Mc Call R.P., Tanner D.B., Miller J.S., Epstein A.J., Howard I.A. and Conwell E.M., Synth. Met. 11 (1985) 231.

[35] Barisic S. and Brazovskii S., in "Recent Developments in Condensed Matter Physics", J.T. Devreese Ed., Vol. 1 (Plenum Press, New York, 1981) p. 327; Emery V.J., Bruinsma R. and Barisic S., Phys. Rev. Lett. 48 (1982) 1039.

[36] a) Penc K. and Mila F., J. Phys. IV France 3 (1993) C2-155: Phys. Rev. B50 (1994) 11429 ; b) Mila F., private communication.

[37] Laversanne R., Coulon C., Gallois B., Pouget J.P. and Moret R., J. Phys. Lett. France 45 (1984) L393.

[38] a) Liu Q., Ravy S., Pouget J.P., Coulon C. and Bourbonna1s C.. Synth. Met. 55-57 (1993) 1840; b) Dumoulin B., Bourbonnais C., Ravy S., Ponget J.P. and Coulon C., Phys Rev. Lett. 76 (1996) 1360. 
[39] Javadi H.H.S., Laversanne R. and Epstein A.J., Phys. Rev. B37 (1988) 4280.

[40] Coulon C.. Parkin S.S.P. and Laversanne R., Phys. Rev. B31 (1985) 3583.

[41] Ilakovac V.. unpublished results.

[42] Granier T., Gallows B., Fritsch A.. Ducasse L. and Coulon C., in "Lower Dimensional Systems and Molecular Devices" NATO-ASI (Plenum Press. 1989). A distinctive feature of (TMTTF) $)_{2} \mathrm{SbF}_{6}$ compared to (TMTTF) ${ }_{2} \mathrm{PF}_{6}$ is the decrease of $t_{\mathrm{s} 1}$ upon cooling (138 meV and $128 \mathrm{meV}$ at $295 \mathrm{~K}$ and $135 \mathrm{~K}$ respectively) in the $\mathrm{SbF}_{6}$ salt and its increase in the $\mathrm{PF}_{6}$ one (Tab. I). Differently $t_{\mathrm{s} 2}(85 \mathrm{meV}$ and $103 \mathrm{meV}$ respectively at $295 \mathrm{~K}$ and $135 \mathrm{~K}$ in the $\mathrm{SbF}_{6}$ salt) increases for both salts upon cooling.

[43] Ilakovac V., Ravy S., Pouget J.P., Riess W., Brutting W. and Schwoerer M.. J. Phys. IV France 3 (1993) C2-137.

[44] Hirsch J.E. and Scalapino D.J., Phys. Rev. B27 (1983) 7169; 29 (1984) 5541; Caron L.G. and Bourbonnais C., Phys. Rev. B29 (1984) 4230.

[45] Caron L.G. and Bourbonnass C., Physica 23B (1986) 453.

[46] Auban-Senzier P., Gotschy B., Farrall A., Bourbonnais C., Jerome D., Henriques R.T., Johannsen I. and Bechgaard K., J. Phys. I France 2 (1992) 677 and earlier references there in.

[47] Liu Q., Thesis (Orsay, 1992).

[48] a) Pouget J.P.. Moret R., Comes R. and Bechgaard K., J. Phys. Lett. France 42 (1981) L543; b) Moret R., Ravy S., Pouget J.P., Comes R. and Bechgaard K., Phys. Rev. Lett. 57 (1986) 1915; c) Leung P.C.W., Schultz A.J., Wang H.H., Emge T.J., Ball G.A., Cox D.D. and Williams J.M., Phys. Rev. B30 (1984) 1615; d) Moret R., Pouget J.P., Comes R. and Bechgaard K., J. Phys. Colloq. France 44 (1983) C3-957; e) Ravy S., Moret R., Pouget J.P. and Comes R., Synth. Met. 13 (1986) 63; f) Ravy S., Pouget J.P., Moret R. and Wudl F., J. Phys. I France 1 (1991) 703; g) Pouget J.P.. Shirane G., Bechgaard K. and Fabre J.M., Phys. Rev. B27 (1983) 5203; h) Parkin S.S.P., Coulon C., Moret and Pouget J.P., Phys. Rev. B 36 (1987) 2246: i) Parkin S.S.P., Mayerle J.J. and Engler E.M., J. Phys. France Colloq. 44 (1983) C3-1105; j) Pouget J.P., Moret R.,Comes R., Bechgaard K.. Fabre J.M. and Giral L., Mol. Cryst. Liq. Cryst. 79 (1982) 129; k) Coulon C., Maaroufi A., Amiell J.. Duppart E., Flandrois S., Delhaes P., Moret R., Pouget J.P. and Morand J.P., Phys. Rev. B 26 (1982) 6322.

[49] a) Moret R., Pouget J.P., Comes R. and Bechgaard K., Phys. Rev. Lett. 49 (1982) 1008; b) Rindorf G., Soling H. and Thorup N., Acta Cryst. C40 (1984) 1137.

[50] Emge T.J., Wang M.H., Beno M.A., Williams J.M., Whangbo M.H. and Evain M., J.A.C.S. 108 (1986) 8215.

[51] a) Moret R., Pouget J.P., Comes R. and Bechgaard K., J. Phys. France 46 (1985) 1521; b) Gallois B., Meresse A.. Gaultier J. and Moret R., Mol. Cryst. Liq. Cryst. 131 (1985) 147.

[52] Guy D.P.R., Boebinger G.S., Marsegria E.A., Friend R.H. and Bechgaard K., J. Phys. C: Solid State Phys. 16 (1983) 691.

[53] a) Gaonach C.. Creuzet G. and Noguera C., J. Phys. France 48 (1987) 107; b) Fertey P. Sayetat F., Muller J., Pouget J.P., Lenorr C. and Batail P., Physica C235-240 (1994) 2459 ; Synth. Met. 70 (1995) 761.

[54] Pouget J.P.. Kagoshima S., Tamegar T., Nogamı Y., Kubo K., Nakajima T. and Bechgaard K., J. Phys. Soc. Jpn 59 (1990) 2036.

[55] Wang W., Hannams S.T. and Chaikin P.M., Phys. Rev. Lett. 70 (1993) 3091.

[56] Chung M.. Figueroa E., Kuo Y.-K.. Wang Y., Brill J.W., Burgin T. and Montgomery L.K., Phys. Rev. B48 (1993) 9256.

[57] Pesty F., Garoche P. and Moradpour A., Mol. Cryst. Liq. Cryst. 119 (1985) 251. 
[58] Pouget J.P., in "Organic and Inorganic Low Dimensional Crystalline Materials" P. Delhaes and M. Drillon Eds., NATO ASI B168 (Plenum Press, 1987) p. 185.

[59] Pouget J.P., Moret R., Comes R., Shirane G., Bechgaard K. and Fabre J.M., J. Phys. France Colloq. 44 (1983) C3-969.

[60] Emery V.J., J. Phys. France Colloq. 44 (1983) C3-977.

[61] Brazovskii S. and Yakovenko V., J. Phys. Lett. 46 (1985) L111; JETP 62 (1985) 1340.

[62] Pouget J.P., in "Low Dimensional Conductors and Superconductors" D. Jerome and L.G. Caron Eds., NATO ASI B155 (Plenum Press, 1987) p.17.

[63] Bruinsma R. and Emery V.J., J. Phys.;France Colloq. 44 (1983) C3-1115.

[64] Tom1c S. and Jerome D., J. Phys.: Cond. Matt. 1 (1989) 4451 and earlier references there in.

[65] Gor'kov L.P. and Lebed A.G., Phys. Rev. B51 (1995) 3285; Synth. Met. 70 (1995) 727.

[66] Ravy S., Moret R., Ponget J.P. and Comes R., Physica 143B (1986) 542.

[67] Ilakovac V., Liu Q., Ravy S., Albouy P.-A., Pouget J.P., Lenolr C. and Batail P., Synth. Met 55-57 (1993) 2372.

[68] Ilakovac V., Ravy S., Boubekeur K., Lenoir C., Batail P. and Pouget J.P. (to be published)

[69] Debye P., Anderson M.R. and Brumberger H., J. Appl. Phys. 28 (1957) 679.

[70] Kogon H.S. and Wallace D.J., J. Phys. A: Math. Gen. 14 (1981) L527.

[71] Ravy S., Moret R., Pouget J.P., Comes R. and Parkin S.S.P., Phys. Rev. B33 (1986) 2049.

[72] Whangbo M.-H., Ren̊ J., Liang W., Canadell E., Pouget J.P., Ravy S., Williams J.M. and Beno M.A., Inorg. Chem. 31 (1992) 4169.

[73] Leung P.C.W., Emge T.J., Beno M.A., Wang H.H., Williams J.M., Petricek V. and Coppens P., J.A.C.S. 107 (1985) 6184.

[74] Ravy S., Moret R. and Pouget J.P., Phys. Rev. B38 (1988) 4469.

[75] Nogamı Y.. Pouget J.P., Ito H., Ishiguro T. and Saito G., Solvd State Commun. 89 (1994) 113.

[76] Pouget J.P. and Ravy S., Proceedings of ICSM'96, Synth. Met. (to be published). 